\title{
The Zografos-Balakrishnan Odd Log-Logistic Generalized Half-Normal Distribution with Mathematical Properties and Simulations
}

\author{
Mahdieh Mozafari ${ }^{1}$, Mahmoud Afshari $^{2, *}$, Morad Alizadeh ${ }^{2}$, Hamid Karamikabir $^{2}$ \\ ${ }^{1}$ Department of Mathematics and Computing, Higher Education Complex of Bam, Iran. ${ }^{2}$ Department of Statistics, Persian Gulf \\ University, Bushehr, Iran.
}

\begin{abstract}
In this paper, A new class of distributions called the Zografos-Balakrishnan odd log-logistic Generalized halfnormal (ZOLL-GHN) family with four parameters is introduced and studied. Useful representations and some mathematical properties of the new family include moments, quantile function, moment Generating function are investigated. The maximum likelihood equations for estimating the parameters based on real data are given. Different methods have been used to estimate its parameters such as maximum likelihood, Least squares, weighted least squares, Crammer-von-Misers, Anderson-Darling and right-tailed Anderson-Darling methods. We assesses the performance of the maximum likelihood estimators in terms of biases and mean squared errors by means of a simulation study. Finally, the usefulness of the family and fitness capability of this model, are illustrated by means of two real data sets.
\end{abstract}

Keywords Zografos-Balakrishnan odd log-logistic family, Generalized half-normal family, Quantile function, Moments, Maximum likelihood, Weighted least square, Bias, Mean square error

AMS 2010 subject classifications 60E05, 62F10, 62G05

DOI: $10.19139 /$ soic.v7i1.649

\section{Introduction}

The statistics literature is filled with hundreds of continuous univariate distributions: see Johnson et al. [19, 20]. Recent developments have been focused to define new families by adding shape parameters to control skewness, kurtosis and tail weights thus providing great flexibility in modeling skewed data in practice, including the twopiece approach introduced by Hansen [18] and the Generalized pioneered by Eugen et al. [14], Cordeiro and de Castro [7], Alexander et al. [2] and Cordeiro et al. [9]. Many subsequent articles apply these techniques to induce skewness into well-known symmetric distributions such as the symmetric Student t. For a review, see Aas and Haff [1].

Cordiro et al. [10] introduced a new family of distribution which called Zografos-Balakrishnan odd log-logisticG. The cdf, pdf and hrf of this family are given by the following:

$$
F_{Z B O L L-G}(x ; \alpha, \beta, \boldsymbol{\tau})=\frac{\gamma(\beta,-\log [1-W(x ; ; \boldsymbol{\tau})])}{\Gamma(\beta)}, \quad x \in \mathbb{R}, \beta>0
$$

where, $W(x ; ; \boldsymbol{\tau})=\frac{G(x ; ; \boldsymbol{\tau})^{\alpha}}{G(x ; ; \boldsymbol{\tau})^{\alpha}+\bar{G}(x ; ; \boldsymbol{\tau})^{\alpha}}, \Gamma(\beta)=\int_{0}^{\infty} t^{\beta-1} \mathrm{e}^{-\mathrm{t}} \mathrm{dt}$ and $\gamma(\beta, z)=\int_{0}^{z} t^{\beta-1} \mathrm{e}^{-t} d t$ are the gamma function and lower incomplete gamma function, respectively.

\footnotetext{
*Correspondence to: Mahmoud Afshari (Email: afshar@pgu.ac.ir). Department of Statistics, Persian Gulf University, Bushehr, Iran.
}

ISSN 2310-5070 (online) ISSN 2311-004X (print)

Copyright (C) 2019 International Academic Press 
Note that for any baseline cdf $G(x ; \boldsymbol{\tau})$, and baseline survival function $\bar{G}(x ; \boldsymbol{\tau})=1-G(x ; \boldsymbol{\tau}),(x \in \mathbb{R}$ and $\boldsymbol{\tau}$ denotes the parameters in the parent $\mathrm{G})$, the equation (1) can be rewritten as Follows:

$$
F_{Z B O L L-G}(x ; \alpha, \beta, \boldsymbol{\tau})=\frac{1}{\Gamma(\beta)} \gamma\left\{\beta,-\log \left[1-\frac{G^{\alpha}(x ; \boldsymbol{\tau})}{G^{\alpha}(x ; \boldsymbol{\tau})+\bar{G}^{\alpha}(x ; \boldsymbol{\tau})}\right]\right\} \alpha>0 .
$$

The model (2) is called the ZBOLL-G distribution with parameters $\alpha$ and $\beta$.

Let $g(x ; \boldsymbol{\tau})=d G(x ; \boldsymbol{\tau}) / d x$ be the baseline probability density function (pdf). The density function corresponding to (2) is given by the following:

$$
f_{Z B O L L-G}(x ; \alpha, \beta, \boldsymbol{\tau})=\frac{\alpha g(x ; \boldsymbol{\tau}) G^{\alpha-1}(x ; \boldsymbol{\tau}) \bar{G}^{\alpha-1}(x ; \boldsymbol{\tau})}{\Gamma(\beta)\left[G^{\alpha}(x ; \boldsymbol{\tau})+\bar{G}^{\alpha}(x ; \boldsymbol{\tau})\right]^{2}}\left\{-\log \left[\frac{\bar{G}^{\alpha}(x ; \boldsymbol{\tau})}{G^{\alpha}(x ; \boldsymbol{\tau})+\bar{G}^{\alpha}(x ; \boldsymbol{\tau})}\right]\right\}^{\beta-1} .
$$

The hazard rate function (hrf) of $X$ is given by the following:

$$
\begin{aligned}
h_{Z B O L L-G}(x ; \alpha, \beta, \boldsymbol{\tau}) & =\frac{\alpha g(x ; \boldsymbol{\tau}) G^{\alpha-1}(x ; \boldsymbol{\tau}) \bar{G}^{\alpha-1}(x ; \boldsymbol{\tau})}{\left[G^{\alpha}(x ; \boldsymbol{\tau})+\bar{G}^{\alpha}(x ; \boldsymbol{\tau})\right]^{2}} \\
& \times \frac{\left\{-\log \left[\frac{\bar{G}^{\alpha}(x ; \boldsymbol{\tau})}{G^{\alpha}(x ; \boldsymbol{\tau})+G^{\alpha}(x ; \boldsymbol{\tau})}\right]\right\}^{\beta-1}}{\Gamma(\beta)-\gamma\left\{\beta,-\log \left[1-\frac{G^{\alpha}(x ; \boldsymbol{\tau})}{G^{\alpha}(x ; \boldsymbol{\tau})+G^{\alpha}(x ; \boldsymbol{\tau})}\right]\right\}} .
\end{aligned}
$$

Not that the ZBOLL family is easily simulated by inverting (2) as follows:

if $V$ has the $\gamma(\beta, 1)$ distribution and $G^{-1}(V)=Q_{G}(V)$ where $Q$ is quantile function, then the solution of the nonlinear equation

$$
X=G^{-1}\left\{\frac{\left(1-\mathrm{e}^{-V}\right)^{\frac{1}{\alpha}}}{\left(1-\mathrm{e}^{-V}\right)^{\frac{1}{\alpha}}+\mathrm{e}^{\frac{-V}{\alpha}}}\right\}
$$

has density (3). The parameters $\alpha$ and $\beta$ have a clear interpretation.

Henceforth, a random variable $X$ with density function (3) is denoted by $X \sim$ ZBOLL-G $(\alpha, \beta, \tau)$. The ZBOLL-G family has the same parameters of the parent $\mathrm{G}$ plus the parameters $\alpha$ and $\beta$. For $\alpha=\beta=1$, it reduces to the baseline $\mathrm{G}$ distribution. For $\alpha=1$, we obtain the gamma-G family (Nadaraja, [22]) and for $\beta=1$, we have the Odd log -logistic-G family ( Gleaton and Lynch, [16]).

Gleaton and Lynch [16] introduced a new family of distribution which called generalized log-logistic G. (for short GLL). The cdf and pdf of this family for any baseline $\operatorname{cdf} G$, are given by the following:

$$
\begin{gathered}
F_{O L L-G}(x ; \alpha, \boldsymbol{\tau})=\frac{G(x ; \boldsymbol{\tau})^{\alpha}}{G(x ; \boldsymbol{\tau})^{\alpha}+\bar{G}(x ; \boldsymbol{\tau})^{\alpha}} \\
f_{O L L-G}(x ; \alpha, \boldsymbol{\tau})=\frac{\alpha g(x ; \alpha, \boldsymbol{\tau}) G(x ; \alpha, \boldsymbol{\tau})^{\alpha-1} \bar{G}(x ; \alpha, \boldsymbol{\tau})^{\alpha-1}}{\left[G(x ; \alpha, \boldsymbol{\tau})^{\alpha}+\bar{G}(x ; \alpha, \boldsymbol{\tau})^{\alpha}\right]^{2}}
\end{gathered}
$$

where $g(x)=\frac{d G(x)}{d x}$ and $\alpha>0$ is shape parameter. Since we can using this family by odd transform from log-logistic family, Alizadeh et.al [3], Cordeiro et.al [11] used odd log-logistic G (for short OLL-G) instead of GLL.

Following the key idea of Zografos and Balakrishnan [28] and Ristic and Balakrishnan [24], we can also interpret (3) in this way: if $X_{U(1)}, X_{U(2)}, \ldots, X_{U(n)}$ are upper record values from a sequence of independent random variables with common pdf

$$
f_{O L L-G}(x ; \alpha, \boldsymbol{\tau})=\frac{\alpha g(x ; \boldsymbol{\tau})\{G(x ; \boldsymbol{\tau})[1-G(x ; \boldsymbol{\tau})]\}^{\alpha-1}}{\left\{G^{\alpha}(x ; \boldsymbol{\tau})+\bar{G}^{\alpha}(x ; \boldsymbol{\tau})\right\}^{2}}
$$


then the pdf of the $n$th upper record value has the pdf (3). The paper is organized as follows: In Section 2, we construct the new family of distributions. Shape characteristics of the probability density and hazard functions of the family are investigated. Useful representations are investigated in section 3. Various statistical properties of the proposed distribution include moments, quantile function and moment generating function are explored in Section 4. Estimation of the model parameters by maximum likelihood is performed in Section 5. Simulation study and applications to real data set illustrate the performance of the new family in Section 6 and Section 7. In Section 8, we offer some concluding remarks.

\section{Zografos-Balakrishnan odd log-logistic generalized half-normal (ZOLL-GHN) distribution}

The Generalized Half Normal(GHN) distribution is introduced by Cooray and Ananda [6]. This density function with shape parameter $\lambda>0$ and scale parameter $\theta>0$ (for $x>0$ ), where $\boldsymbol{\tau}=(\lambda, \theta)^{T}$, (Cooray and Ananda, [6]) is the following:

$$
g(x ; \boldsymbol{\tau})=\sqrt{\frac{2}{\pi}}\left(\frac{\lambda}{x}\right)\left(\frac{x}{\theta}\right)^{\lambda} \exp \left[-\frac{1}{2}\left(\frac{x}{\theta}\right)^{2 \lambda}\right] .
$$

It's cumulative distribution function (cd) depends on the error function

$$
G(x ; \boldsymbol{\tau})=2 \Phi\left[\left(\frac{x}{\theta}\right)^{\lambda}\right]-1=\operatorname{erf}\left[\frac{\left(\frac{x}{\theta}\right)^{\lambda}}{\sqrt{2}}\right],
$$

where,

$$
\Phi(x)=\frac{1}{2}\left[1+\operatorname{erf}\left(\frac{x}{\sqrt{2}}\right)\right], \quad \operatorname{erf}(x)=\frac{2}{\sqrt{\pi}} \int_{0}^{x} e^{-t^{2}} d t .
$$

By using (8) and (9) in equations (3) and (2), the density function and cd of ZOLL-GHN(for $x>0$ ), with four parameters $\lambda>0, \theta>0, \alpha>0$ and $\beta>0$, are given by the following:

$$
\begin{gathered}
f(x)=\frac{\alpha \sqrt{\frac{2}{\pi}}\left(\frac{\lambda}{x}\right)\left(\frac{x}{\theta}\right)^{\lambda} \exp \left[-\frac{1}{2}\left(\frac{x}{\theta}\right)^{2 \lambda}\right]\left\{2 \Phi\left[\left(\frac{x}{\theta}\right)^{\lambda}\right]-1\right\}^{\alpha-1}\left\{2-2 \Phi\left[\left(\frac{x}{\theta}\right)^{\lambda}\right]\right\}^{\alpha-1}}{\Gamma(\beta)\left\{\left[2 \Phi\left[\left(\frac{x}{\theta}\right)^{\lambda}\right]-1\right]^{\alpha}+\left[2-2 \Phi\left[\left(\frac{x}{\theta}\right)^{\lambda}\right]\right]^{\alpha}\right\}^{2}} \\
\times\left\{-\log \left[\frac{\left\{2-2 \Phi\left[\left(\frac{x}{\theta}\right)^{\lambda}\right]\right\}^{\alpha}}{\left.\left.\left\{2 \Phi\left[\left(\frac{x}{\theta}\right)^{\lambda}\right]-1\right\}^{\alpha}+\left\{2-2 \Phi\left[\left(\frac{x}{\theta}\right)^{\lambda}\right]\right\}^{\alpha}\right]\right\}^{\beta-1},}\right.\right. \\
F(x)=\frac{1}{\Gamma(\beta)} \gamma\left\{\beta,-\log \left[1-\frac{\left\{2 \Phi\left[\left(\frac{x}{\theta}\right)^{\lambda}\right]-1\right\}^{\alpha}}{\left\{2 \Phi\left[\left(\frac{x}{\theta}\right)^{\lambda}\right]-1\right\}^{\alpha}+\left\{2-2 \Phi\left[\left(\frac{x}{\theta}\right)^{\lambda}\right]\right\}^{\alpha}}\right]\right\},
\end{gathered}
$$

respectively. Also, href of $X$ is given by the following:

$$
\begin{gathered}
h(x)=\frac{\alpha \sqrt{\frac{2}{\pi}}\left(\frac{\lambda}{x}\right)\left(\frac{x}{\theta}\right)^{\lambda} \exp \left[-\frac{1}{2}\left(\frac{x}{\theta}\right)^{2 \lambda}\right]\left\{2 \Phi\left[\left(\frac{x}{\theta}\right)^{\lambda}\right]-1\right\}^{\alpha-1}\left\{2-2 \Phi\left[\left(\frac{x}{\theta}\right)^{\lambda}\right]\right\}^{\alpha-1}}{\left\{\left[2 \Phi\left[\left(\frac{x}{\theta}\right)^{\lambda}\right]-1\right]^{\alpha}+\left[2-2 \Phi\left[\left(\frac{x}{\theta}\right)^{\lambda}\right]\right]^{\alpha}\right\}^{2}} \\
\times \frac{\left\{-\log \left[\frac{\left\{2-2 \Phi\left[\left(\frac{x}{\theta}\right)^{\lambda}\right]\right\}^{\alpha}}{\left.\left.\left\{2 \Phi\left[\left(\frac{x}{\theta}\right)^{\lambda}\right]-1\right\}^{\alpha}+\left\{2-2 \Phi\left[\left(\frac{x}{\theta}\right)^{\lambda}\right]\right\}^{\alpha}\right]\right\}^{\beta-1}}\right.\right.}{\Gamma(\beta)-\gamma\left\{\beta,-\log \left[1-\frac{\left\{2 \Phi\left[\left(\frac{x}{\theta}\right)^{\lambda}\right]-1\right\}^{\alpha}}{\left\{2 \Phi\left[\left(\frac{x}{\theta}\right)^{\lambda}\right]-1\right\}^{\alpha}+\left\{2-2 \Phi\left[\left(\frac{x}{\theta}\right)^{\lambda}\right]\right\}^{\alpha}}\right]\right\}} .
\end{gathered}
$$

In Figure 1 some pdf for EGGO have been drown and In Figure 2 some distribution function and hazard functions for EGGO have been drown. 


\section{Useful representations}

The Generalized binomial coefficient for real arguments is given by $\left(\begin{array}{l}x \\ y\end{array}\right)=\Gamma(x+1) /[\Gamma(y+1) \Gamma(x-y+1)]$. By using the incomplete gamma function expansion, we can write

$$
F(x)=\frac{1}{\Gamma(\beta)} \sum_{i=0}^{\infty} \frac{(-1)^{i}}{i !(\beta+i)}\left\{-\log \left[1-\frac{G(x)^{\alpha}}{G(x)^{\alpha}+\bar{G}(x)^{\alpha}}\right]\right\}^{\beta+i} .
$$

For any real positive power parameter, the formula below holds (http://functions.wolfram.com/ElementaryFunctions/Log/06/01/04/03/)

$$
\begin{aligned}
\left\{-\log \left[1-\frac{G(x)^{\alpha}}{G(x)^{\alpha}+\bar{G}(x)^{\alpha}}\right]\right\}^{\beta+i} & =(\beta+i) \sum_{k=0}^{\infty} \sum_{j=0}^{k} \frac{(-1)^{j+k}\left(\begin{array}{c}
k-\beta-i \\
k
\end{array}\right)\left(\begin{array}{l}
k \\
j
\end{array}\right)}{(\beta+i-j)} \\
& \times p_{j, k}\left[\frac{G(x)^{\alpha}}{G(x)^{\alpha}+\bar{G}(x)^{\alpha}}\right]^{\beta+i+k},
\end{aligned}
$$

where the constants $p_{j, k}$ can be determined recursively by the following:

$$
p_{j, k}=k^{-1} \sum_{m=1}^{k}[k-m(j+1)] c_{m} p_{j, k-m}
$$

for $k=1,2, \ldots, c_{k}=(-1)^{k+1} /(k+1)$ and $p_{j, 0}=1$.

Further,

$$
\left[\frac{G(x)^{\alpha}}{G(x)^{\alpha}+\bar{G}(x)^{\alpha}}\right]^{\beta+i+k}=\frac{\sum_{r=0}^{\infty} \lambda_{r} G(x)^{r}}{\sum_{r=0}^{\infty} \rho_{r} G(x)^{r}}=\sum_{r=0}^{\infty} a_{r} G(x)^{r}
$$
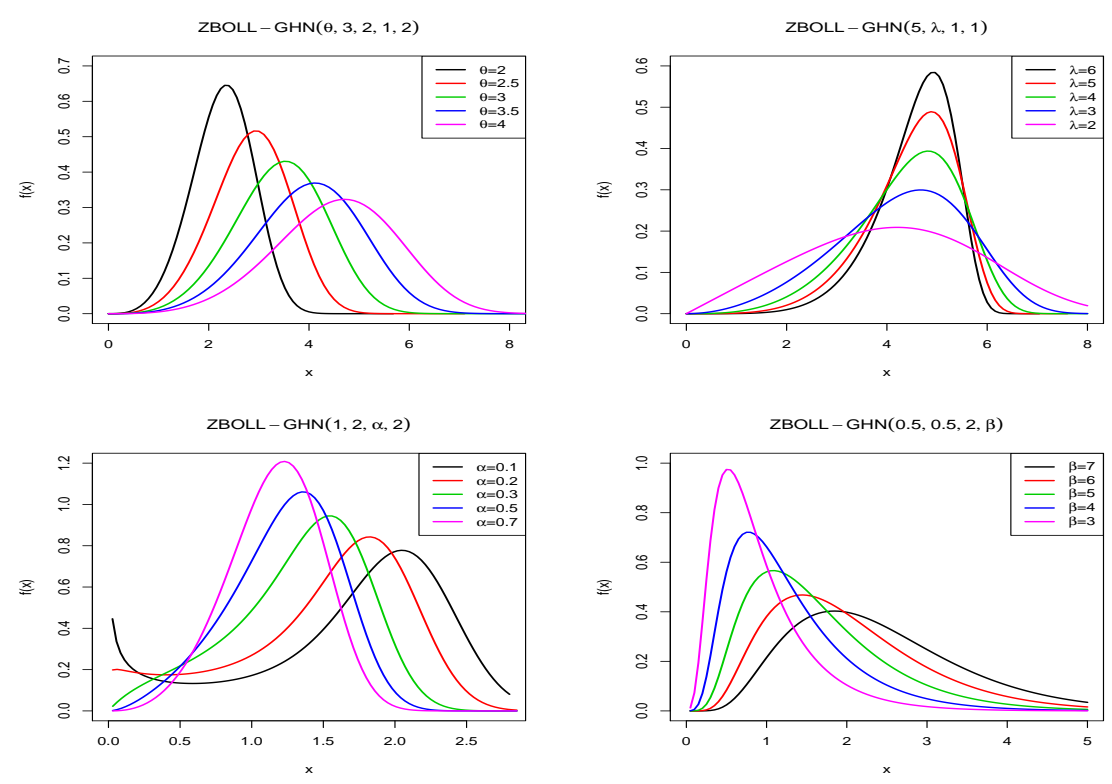

Figure 1. Plots of the ZBOLL-GHN $(\theta, \lambda, \alpha, \beta)$ density function for some parameter values. 

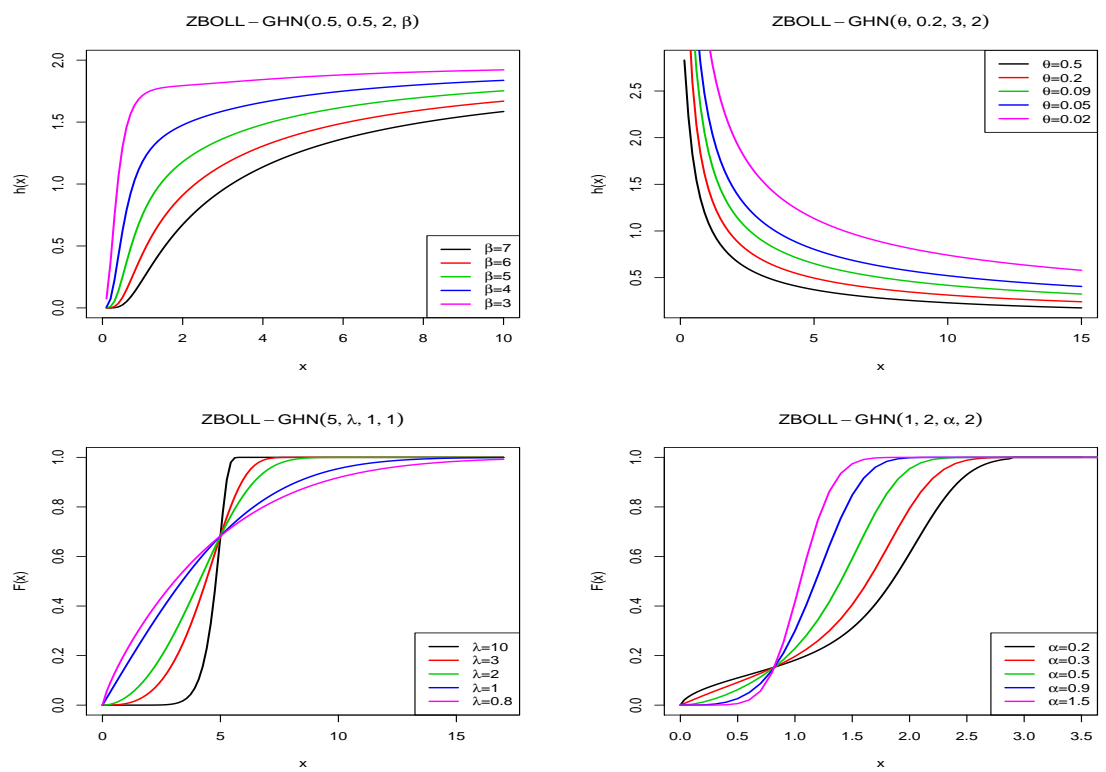

Figure 2. Plots of the ZBOLL-GHN $(\theta, \lambda, \alpha, \beta)$ hazard ratio function and Distribution function for some parameter values.

where,

$$
\lambda_{r}=\sum_{l=r}^{\infty}(-1)^{l+r}\left(\begin{array}{c}
\alpha(\beta+i+k) \\
l
\end{array}\right)\left(\begin{array}{l}
l \\
r
\end{array}\right), \rho_{r}=h_{r}(\alpha, \beta+i+k),
$$

and (for $r \geq 1$ ),

$$
a_{r}=a_{r}(\alpha, \beta, i, k)=\frac{1}{\rho_{0}}\left(\rho_{r}-\frac{1}{\rho_{0}} \sum_{s=1}^{r} \rho_{s} a_{r-s}\right) .
$$

Note that $a_{0}=\lambda_{0} / \rho_{0}$ and $h_{r}(\alpha, \beta+i+k)$ is defined in Appendix A.

Then, equation (2) can be expressed as follows:

$$
F(x)=\sum_{r=0}^{\infty} b_{r} H_{r}(x)
$$

where,

$$
b_{r}=\frac{1}{\Gamma(\beta)} \sum_{i, k=0}^{\infty} \sum_{j=0}^{k} \frac{(-1)^{i+j+k} p_{j, k} a_{r}(\alpha, \beta, i, k)}{(\beta+i-j) i !}\left(\begin{array}{c}
k-\beta-i \\
k
\end{array}\right)\left(\begin{array}{l}
k \\
j
\end{array}\right),
$$

and $H_{r}(x)$ denotes the cd of the exponentiated Generalized half-normal density function with parameter (r) (exp$G H N(r)$ ), is given by the following:

$$
H_{r}(x)=\left\{2 \Phi\left[\left(\frac{x}{\theta}\right)^{\lambda}\right]-1\right\}^{r}
$$

The pdf (10) reduces to the following:

$$
f(x)=\sum_{r=0}^{\infty} b_{r+1} h_{r+1}(x)
$$


where,

$$
h_{r+1}(x)=(r+1) \sqrt{\frac{2}{\pi}}\left(\frac{\lambda}{x}\right)\left(\frac{x}{\theta}\right)^{\lambda} \exp \left[-\frac{1}{2}\left(\frac{x}{\theta}\right)^{2 \lambda}\right]\left\{2 \Phi\left[\left(\frac{x}{\theta}\right)^{\lambda}\right]-1\right\}^{r}
$$

\section{Statistical properties}

In this section some statistical properties of the proposed distribution are investigated.

\subsection{Moments}

Lemma 1

Let $X \sim Z B O L L-G H N(\alpha, \beta, \lambda, \theta)$, then we can write as follows:

$$
E\left(X^{n}\right)=\theta^{n} \sqrt{\frac{2}{\pi}} \sum_{i=1}^{n} B_{r} F_{A}^{(r)}\left(\frac{r+\frac{n}{\lambda}+1}{2} ; \frac{1}{2}, \ldots, \frac{1}{2} ; \frac{3}{2}, \ldots, \frac{3}{2} ;-1, \ldots,-1\right),
$$

where,

$$
B_{r}=\pi^{-\frac{r}{2}} 2^{2 r+\frac{n}{2 \lambda}-\frac{1}{2}} \Gamma\left(\frac{r+\frac{n}{\lambda}+1}{2}\right) b_{r+1}
$$

Proof

We have from equation (16)

$$
\begin{aligned}
E\left(X^{n}\right) & =\sum_{i=1}^{n} b_{r+1} \int_{0}^{\infty} h_{r+1}(x) d x \\
& =\sqrt{\frac{2}{\pi}} \sum_{r=0}^{\infty}(r+1) b_{r+1} \int_{0}^{\infty} x^{n}\left(\frac{\lambda}{x}\right)\left(\frac{x}{\theta}\right)^{\lambda} \exp \left[-\frac{1}{2}\left(\frac{x}{\theta}\right)^{2 \lambda}\right]\left\{2 \Phi\left[\left(\frac{x}{\theta}\right)^{\lambda}\right]-1\right\}^{r} d x
\end{aligned}
$$

By setting $u=\left(\frac{x}{\theta}\right)^{\lambda}$ and considering the error function as the cd of the GHN distribution, the $n$th moment of $X$ can be obtained as the following:

$$
E\left(X^{n}\right)=\theta^{n} \sqrt{\frac{2}{\pi}} \sum_{r=0}^{\infty}(r+1) b_{r+1} I\left(\frac{n}{\lambda}, r\right)
$$

where,

$$
I\left(\frac{n}{\lambda}, r\right)=\int_{0}^{\infty} u^{\frac{n}{\lambda}} \exp \left(-\frac{u^{2}}{2}\right)\left[\operatorname{erf}\left(\frac{u}{\sqrt{2}}\right)\right]^{r} d u .
$$

Inserting the power series for the error function:

$$
\operatorname{erf}(x)=\frac{2}{\sqrt{\pi}} \sum_{m=0}^{\infty} \frac{(-1)^{m} x^{2 m+1}}{(2 m+1) m !}
$$

we obtain as the following:

$$
I\left(\frac{n}{\lambda}, r\right)=\pi^{-\frac{r}{2}} 2^{r+\frac{n}{2 \lambda}-\frac{1}{2}} \sum_{m_{1}, \ldots, m_{r}=0}^{\infty} \frac{(-1)^{m_{1}+\ldots+m_{r}} \Gamma\left(m_{1}+\ldots+m_{r}+\frac{r+\frac{n}{\lambda}+1}{2}\right)}{\left(m_{1}+\frac{1}{2}\right) \ldots\left(m_{r}+\frac{1}{2}\right) m_{1} ! \ldots m_{r} !} .
$$


Further, for the very special case when $r+\frac{n}{\lambda}$ is even, the integral $I\left(\frac{n}{\lambda}, r\right)$ can be expressed in terms of the Varicella function of type A defined by follows:

$$
\begin{aligned}
F_{A}^{(n)}\left(a ; b_{1}, \ldots, b_{n} ; c_{1}, \ldots, c_{n} ; x_{1}, \ldots, x_{n}\right) & =\sum_{m_{1}=0}^{\infty} \ldots \sum_{m_{n}=0}^{\infty} \frac{(a)_{m_{1}+\ldots+m_{n}}\left(b_{1}\right)_{m_{1}} \ldots\left(b_{n}\right)_{m_{n}}}{\left(c_{1}\right)_{m_{1}} \ldots\left(c_{n}\right)_{m_{n}}} \\
& \times \frac{x_{1}^{m_{1}} \ldots x_{n}^{m_{n}}}{m_{1} ! \ldots m_{n} !}
\end{aligned}
$$

where $(a)_{k}=a(a+1) \ldots(a+k-1)$ is the ascending factorial (with the convention that $\left.(a)_{0}=1\right)$. Numerical routines for the direct computation of the Varicella function of type A are available, see Extol [15] and Mathematica (Trott, [27]). Hence, $E\left(X^{n}\right)$ can be expressed in terms of the Varicella functions of type A as the following:

$$
E\left(X^{n}\right)=\theta^{n} \sqrt{\frac{2}{\pi}} \sum_{i=1}^{n} B_{r} F_{A}^{(r)}\left(\frac{r+\frac{n}{\lambda}+1}{2} ; \frac{1}{2}, \ldots, \frac{1}{2} ; \frac{3}{2}, \ldots, \frac{3}{2} ;-1, \ldots,-1\right) .
$$

\subsection{Quantile function}

The gamma regularized function is defined by $Q(\beta, z)=\int_{z}^{\infty} x^{\beta-1} \mathrm{e}^{-\mathrm{x}} / \Gamma(\beta) \mathrm{dx}$. The inverse gamma regularized function $Q^{-1}(\beta, u)$ admits a power series expansion given by the following:

$$
Q^{-1}(\beta, u)=w \sum_{i=0}^{\infty} m_{i} w^{i}
$$

where $w=[\Gamma(\beta+1)(1-u)]^{1 / \beta}, m_{0}=1, m_{1}=1 /(\beta+1), m_{2}=(3 \beta+5) /\left[2(\beta+1)^{2}(\beta+2)\right], m_{3}=[\beta(8 \beta+$ $3)+31] /\left[2(\beta+1)^{3}(\beta+2)(\beta+3)\right]$, etc.

(see: http://functions.wolfram.com/GammaBetaErf/InverseGammaRegulari zed/06/01/03/).

First we consider,

$$
B=\frac{\left(1-\mathrm{e}^{-\mathrm{v}}\right)^{\frac{1}{\beta}}}{\left(1-\mathrm{e}^{-\mathrm{v}}\right)^{\frac{1}{\beta}}+\mathrm{e}^{\frac{-\mathrm{v}}{\beta}}}=\frac{1}{1+\mathrm{e}^{\frac{-\mathrm{v}}{\beta}}\left(1-\mathrm{e}^{-\mathrm{v}}\right)^{\frac{-1}{\beta}}}
$$

By using Taylor expansion and Generalized binomial expansion, we have obtain

$$
\mathrm{e}^{\frac{-\mathrm{v}}{\beta}}\left(1-\mathrm{e}^{-\mathrm{v}}\right)^{\frac{-1}{\beta}}=\sum_{\mathrm{k}=0}^{\infty} \mathrm{b}_{\mathrm{k}}^{*} \mathrm{v}^{\mathrm{k}}
$$

where $b_{0}^{*}=1$ and, for $k \geq 1, b_{k}^{*}=\sum_{j=0}^{\infty} \frac{(-1)^{j+k}\left(j+\beta^{-1}\right)^{k}}{k !}\left(\begin{array}{c}-1 / \beta \\ j\end{array}\right)$.

Then,

$$
B=\frac{1}{\sum_{k=0}^{\infty} b_{k}^{*} v^{k}}=\sum_{k=0}^{\infty} c_{k}^{*} v^{k}
$$

where $c_{0}^{*}=1 / b_{0}^{*}$ and $c_{k}^{*}$ (for $k \geq 1$ ) is obtained from the last equation as

$$
c_{k}^{*}=-\frac{1}{b_{0}^{*}} \sum_{r=1}^{k} b_{r}^{*} c_{k-r}^{*}
$$


Further, we can write as follows:

$$
\begin{aligned}
A & =\frac{\left(1-\mathrm{e}^{-\mathrm{Q}^{-1}(\beta, \mathrm{u})}\right)^{\frac{1}{\beta}}}{\left(1-\mathrm{e}^{-\mathrm{Q}^{-1}(\beta, \mathrm{u})}\right)^{\frac{1}{\beta}}+\mathrm{e}^{\frac{-\mathrm{Q}^{-1}(\beta, \mathrm{u})}{\beta}}}=\sum_{k=0}^{\infty} c_{k}^{*}\left[Q^{-1}(\beta, u)\right]^{k} \\
& =\sum_{k=0}^{\infty} c_{k}^{*}\left(u \sum_{i=0}^{\infty} m_{i} u^{i}\right)^{k} .
\end{aligned}
$$

We use an equation by Gradshteyn and Ryzhik [17] for a power series raised to a positive integer $j$ as follows:

$$
\left(\sum_{i=0}^{\infty} a_{i} u^{i}\right)^{j}=\sum_{i=0}^{\infty} c_{j, i} u^{i}
$$

Here, for $j \geq 0, c_{j, 0}=a_{0}^{j}$, and the coefficients $c_{j, i}$ (for $i=1,2, \ldots$ ) are determined from the recurrence equation

$$
c_{j, i}=\left(i a_{0}\right)^{-1} \sum_{p=1}^{i}[p(j+1)-i] a_{p} c_{j, i-p},
$$

So, the coefficient $c_{j, i}$ follows from $c_{j, 0}, \ldots, c_{j, i-1}$ and then from $a_{0}, \ldots, a_{i}$.

Based on equations (19) and (20), we can rewrite (18) as the following:

$$
A=\sum_{i, k=0}^{\infty} c_{k}^{*} v_{k, i} u^{i+k}=\sum_{l=0}^{\infty} d_{l}^{*} u^{l},
$$

where, for $k \geq 0$, the coefficients $v_{k, i}$ (for $i=1,2, \ldots$ ) are determined from the recurrence equation

$$
v_{k, i}=\left(i m_{0}\right)^{-1} \sum_{p=1}^{i}[p(j+1)-i] m_{p} v_{k, i-p},
$$

with $v_{k, 0}=m_{0}^{k}$ and $d_{l}^{*}=\sum_{(i, k) \in I_{l}} c_{k}^{*} v_{k, i}$ and $I_{l}=\{(i, k) \mid i+k=l ; i, k=0,1,2, \ldots\}$. Then, the quantize function (qt) of $X$ reduces to

$$
Q(u)=Q_{G H N}\left(\sum_{l=0}^{\infty} d_{l}^{*} u^{l}\right) .
$$

In General, even when $Q_{G}(u)$ does not have a closed-form expression, this function can usually be expressed in terms of a power series

$$
Q_{G}(u)=\sum_{i=0}^{\infty} s_{i} u^{i}
$$

where the coefficients $s_{i}$ 's are suitably chosen real numbers. For several important distributions, such as the normal, Student $t$, gamma and beta distributions, $Q_{G}(u)$ does not have a closed-form expression but it can be expanded as in equation (22).

The qt of the GHN distribution can be obtained as the following:

$$
Q_{G H N}=\theta\left[\Phi^{-1}\left(\frac{1+u}{2}\right)\right]^{\frac{1}{\lambda}}
$$


Using equation (22) and the generalized binomial expansion, we can write as follows:

$$
\Phi^{-1}\left(\frac{1+u}{2}\right)=\sum_{i=0}^{\infty} \sum_{j=0}^{i} \frac{a_{j}\left(\begin{array}{l}
i \\
j
\end{array}\right)}{2^{i}} u^{j}=\sum_{k=0}^{\infty} \delta_{k} u^{k},
$$

where $\delta_{k}=\sum_{i=0}^{\infty} \sum_{j=0}^{i} \frac{a_{j}\left(\begin{array}{l}i \\ j\end{array}\right)}{2^{i}}$. Based on equation (30) given in Appendix A, we obtain

$$
\left[\sum_{k=0}^{\infty} \delta_{k} u^{k}\right]^{\frac{1}{\lambda}}=\sum_{l=0}^{\infty} b_{l}\left[\sum_{k=0}^{\infty} \delta_{k} u^{k}\right]^{l}
$$

where $b_{l}=\sum_{s=l}^{\infty} \sum_{j=0}^{i} \frac{(-1)^{l}\left(\frac{1}{\lambda}\right)_{s}}{s !}\left(\begin{array}{l}s \\ l\end{array}\right)$ and $\left(\frac{1}{\lambda}\right)_{s}=\left(\frac{1}{\lambda}\right)\left(\frac{1}{\lambda}-1\right) \ldots\left(\frac{1}{\lambda}-s+1\right)$ is the falling factorial.

Further, by using equations (19) and (20)we obtain as follows:

$$
\left[\sum_{k=0}^{\infty} \delta_{k} u^{k}\right]^{\frac{1}{\lambda}}=\sum_{l=0}^{\infty} b_{l}\left[\sum_{n=0}^{\infty} \delta_{n} u^{n}\right]^{l}=\sum_{l=0}^{\infty} b_{l} \sum_{n=0}^{\infty} d_{l, n} u^{n}=\sum_{n=0}^{\infty} e_{n} u^{n}
$$

where the equation $d_{l, n}=\left(n \delta_{0}\right)^{-1} \sum_{m=1}^{n}[m(n+1)-j] \delta_{m} d_{l, n-m}$ (for $\left.n \geq 1\right), d_{l, 0}=\delta_{0}^{k}$ and $e_{n}=\sum_{l=0}^{\infty} b_{l} d_{l, n}$.

We can write the qt of GHN distribution as the power series

$$
Q_{G H N}(u)=\theta \sum_{n=0}^{\infty} e_{n} u^{n} .
$$

By combining equations (21) and (24) and using equations (19) and (20), we obtain as follows:

$$
Q(u)=\theta \sum_{l=0}^{\infty} h_{l} u^{l}
$$

where $h_{l}=\sum_{n=0}^{\infty} e_{n} h_{n, l}$ (for $n \geq 0$ and $l \geq 0$ ), $h_{n, l}=\left(l d_{0}^{*}\right)^{-1} \sum_{p=1}^{l}[p(n+1)-l] d_{p}^{*} h_{n, l-p}$, for $l \geq 1$, and $h_{l, 0}=d_{0}^{*}$.

Hence, equation (25) reveals that the qt of the ZBOLL-GHN distribution can be expressed as a power series. For practical purposes, we can adopt ten terms in this power series.

Let $W(\cdot)$ be any integrable function in the positive real line. We can write as follows:

$$
\int_{-\infty}^{\infty} W(x) f(x) d x=\int_{0}^{1} W\left(\sum_{l=0}^{\infty} h_{l} u^{l}\right) d u .
$$

Equations (25) and (26) are the main results of this section. We can obtain from them various ZOLL-GHN mathematical properties using integrals over $(0,1)$, which are usually more simple than if they are based on the left integral.

\subsection{Moment generating function}

The moment Generating function (mf) $M(t)$ of a random variable $X$ provides the basis of an alternative route to analytical results compared with working directly with the pdf and cdf of $X$.

Lemma 2

Let $X \sim Z B O L L-G H N(\alpha, \beta, \lambda, \theta)$, then

$$
M(t)=\sum_{r, l=0}^{\infty} \frac{\theta^{r} v_{r, l}}{(l+1) r !} t^{r}
$$

where $v_{r, 0}=e_{0}^{r}$ and for $n \geq 1, v_{r, l}=\left(l e_{0}\right)^{-1} \sum_{m=1}^{r}[m(l+1)-l] e_{m} v_{l, r-m}$. 
Proof

We can write from equations (19) and (20)

$$
\begin{aligned}
M(t) & =\int_{0}^{1} \exp [t Q(u)] d u=\int_{0}^{1} \exp \left[t \theta \sum_{l=0}^{\infty} h_{l} u^{l}\right] d u \\
& =\sum_{r=0}^{\infty} \int_{0}^{1} \frac{(t \theta)^{r}\left(\sum_{l=0}^{\infty} h_{l} u^{l}\right)^{r}}{r !} d u=\sum_{r, l=0}^{\infty} \frac{\theta^{r} v_{r, l}}{(l+1) r !} t^{r}
\end{aligned}
$$

Clearly for the $k$ th moment of $X$ follows from the last equation as $\mu_{k}^{\prime}=\sum_{l=0}^{\infty} \frac{\theta^{r} v_{r, l}}{(l+1)}$.

\section{Maximum likelihood estimation}

In this section, we determine the maximum likelihood estimates (MLE's) of the model parameters of the new family from complete samples only. Let $x_{1}, \ldots, x_{n}$ be observed values from the ZOLL-GHN family with parameters $\alpha$, $\beta, \lambda$ and $\theta$. Let $\Theta=(\alpha, \beta, \lambda, \theta)^{\top}$ be the $r \times 1$ parameter vector. The total log-likelihood function for $\theta$ is given by

$$
\begin{aligned}
\ell_{n}(\Theta) & =\ell_{n}=n \log (\alpha)-n \log [\Gamma(\beta)]+n \log \left(\sqrt{\frac{2}{\pi}}\right)+n \log (\lambda)-n \lambda \log (\theta) \\
& +(\lambda-1) \sum_{i=1}^{n} \log \left(x_{i}\right)-\frac{1}{2} \sum_{i=1}^{n}\left(\frac{x_{i}}{\theta}\right)^{2 \lambda}+(\alpha-1) \sum_{i=1}^{n} \log \left(q_{i}-1\right) \\
& +(\alpha-1) \sum_{i=1}^{n} \log \left(2-q_{i}\right)-2 \sum_{i=1}^{n} \log \left\{\left(q_{i}-1\right)^{\alpha}+\left(2-q_{i}\right)^{\alpha}\right\} \\
& +(\beta-1) \sum_{i=1}^{n} \log \left\{-\log \left[\frac{\left(2-q_{i}\right)^{\alpha}}{\left(q_{i}-1\right)^{\alpha}+\left(2-q_{i}\right)^{\alpha}}\right]\right\}
\end{aligned}
$$

where $x_{i}>0$ and $q_{i}=2 \Phi\left[\left(\frac{x_{i}}{\theta}\right)^{\lambda}\right]$.

The log-likelihood function can be maximized either directly by using the ASS (PROC UNMIXED) or by solving the nonlinear likelihood equations obtained by differentiating (28). The components of the score function 
$U_{n}(\Theta)=\left(\partial \ell_{n} / \partial \alpha, \partial \ell_{n} / \partial \beta, \partial \ell_{n} / \partial \lambda, \partial \ell_{n} / \partial \theta\right)^{\top}$ are given by the following:

$$
\begin{aligned}
& \frac{\partial \ell_{n}}{\partial \alpha}=\frac{n}{\alpha}+\sum_{i=1}^{n} \log \left(q_{i}-1\right)+\sum_{i=1}^{n} \log \left(2-q_{i}\right) \\
& -2 \sum_{i=1}^{n} \frac{\left(q_{i}-1\right)^{\alpha} \log \left(q_{i}-1\right)+\left(2-q_{i}\right)^{\alpha} \log \left(2-q_{i}\right)}{\left(q_{i}-1\right)^{\alpha}+\left(2-q_{i}\right)^{\alpha}} \\
& +(\beta-1) \sum_{i=1}^{n} \frac{\left(q_{i}-1\right)^{\alpha} \log \left(\frac{q_{i}-1}{2-q_{i}}\right)}{\left[\left(q_{i}-1\right)^{\alpha}+\left(2-q_{i}\right)^{\alpha}\right] \log \left\{\frac{\left(2-q_{i}\right)^{\alpha}}{\left(q_{i}-1\right)^{\alpha}+\left(2-q_{i}\right)^{\alpha}}\right\}}, \\
& \frac{\partial \ell_{n}}{\partial \beta}=-n \psi(\beta)+\sum_{i=1}^{n} \log \left\{-\log \left[\frac{\left(2-q_{i}\right)^{\alpha}}{\left(q_{i}-1\right)^{\alpha}+\left(2-q_{i}\right)^{\alpha}}\right]\right\} \text {, } \\
& \frac{\partial \ell_{n}}{\partial \lambda}=\frac{n}{\lambda}-n \log (\theta)+\sum_{i=1}^{n} \log \left(x_{i}\right)-\sum_{i=1}^{n}\left(\frac{x_{i}}{\theta}\right)^{2 \lambda} \log \left(\frac{x_{i}}{\theta}\right) \\
& +(\alpha-1) \sum_{i=1}^{n} \frac{q_{i}^{(\lambda)}}{q_{i}-1}-(\alpha-1) \sum_{i=1}^{n} \frac{q_{i}^{(\lambda)}}{2-q_{i}} \\
& -2 \alpha \sum_{i=1}^{n} \frac{q_{i}^{(\lambda)}\left[\left(q_{i}-1\right)^{\alpha-1}-\left(2-q_{i}\right)^{\alpha-1}\right]}{\left(q_{i}-1\right)^{\alpha}+\left(2-q_{i}\right)^{\alpha}} \\
& -\alpha(\beta-1) \sum_{i=1}^{n} \frac{q_{i}^{(\lambda)}\left(2-q_{i}\right)^{-1}\left(q_{i}-1\right)^{\alpha-1}}{\left[\left(q_{i}-1\right)^{\alpha}+\left(2-q_{i}\right)^{\alpha}\right] \log \left\{\frac{\left(2-q_{i}\right)^{\alpha}}{\left(q_{i}-1\right)^{\alpha}+\left(2-q_{i}\right)^{\alpha}}\right\}}, \\
& \frac{\partial \ell_{n}}{\partial \theta}=-\frac{n \lambda}{\theta}+\frac{\lambda}{\theta} \sum_{i=1}^{n}\left(\frac{x_{i}}{\theta}\right)^{2 \lambda}+(\alpha-1) \sum_{i=1}^{n} \frac{q_{i}^{(\theta)}}{q_{i}-1} \\
& -(\alpha-1) \sum_{i=1}^{n} \frac{q_{i}^{(\theta)}}{2-q_{i}}-2 \alpha \sum_{i=1}^{n} \frac{q_{i}^{(\theta)}\left[\left(q_{i}-1\right)^{\alpha-1}-\left(2-q_{i}\right)^{\alpha-1}\right]}{\left(q_{i}-1\right)^{\alpha}+\left(2-q_{i}\right)^{\alpha}} \\
& -\alpha(\beta-1) \sum_{i=1}^{n} \frac{q_{i}^{(\theta)}\left(2-q_{i}\right)^{-1}\left(q_{i}-1\right)^{\alpha-1}}{\left[\left(q_{i}-1\right)^{\alpha}+\left(2-q_{i}\right)^{\alpha}\right] \log \left\{\frac{\left(2-q_{i}\right)^{\alpha}}{\left(q_{i}-1\right)^{\alpha}+\left(2-q_{i}\right)^{\alpha}}\right\}},
\end{aligned}
$$

where,

$$
\begin{gathered}
q_{i}^{(\lambda)}=\frac{\partial q_{i}}{\partial \lambda}=2\left(\frac{x_{i}}{\theta}\right)^{\lambda} \log \left(\frac{x_{i}}{\theta}\right) \phi\left[\left(\frac{x_{i}}{\theta}\right)^{\lambda}\right], \\
q_{i}^{(\theta)}=\frac{\partial q_{i}}{\partial \theta}=-2\left(\frac{\lambda}{\theta}\right)\left(\frac{x_{i}}{\theta}\right)^{\lambda} \phi\left[\left(\frac{x_{i}}{\theta}\right)^{\lambda}\right],
\end{gathered}
$$

and $\psi(\cdot)$ is the $\digamma$ function. The MLE $\widehat{\Theta}$ of $\Theta$ is obtained by solving the nonlinear likelihood equations $U_{\alpha}(\Theta)=0$, $U_{\beta}(\Theta)=0, U_{\lambda}(\Theta)=0$ and $U_{\theta}(\Theta)=0$. These equations cannot be solved analytically and statistical software can be used to solve them numerically. We can use iterative techniques such as a Newton-Raison type algorithm to obtain the estimate $\widehat{\Theta}$. We employ the numerical procedure Unmixed in ASS.

For interval estimation of $(\alpha, \beta, \lambda, \theta)$ and hypothesis tests on these parameters, we obtain the observed information matrix since the expected information matrix is very complicated and requires numerical integration. The $4 \times 4$ observed information matrix $J(\Theta)$, becomes as follows:

$$
J(\Theta)=-\left(\begin{array}{cccc}
\mathbf{L}_{\alpha \alpha} & \mathbf{L}_{\alpha \beta} & \mathbf{L}_{\alpha \lambda} & \mathbf{L}_{\alpha \theta} \\
\cdot & \mathbf{L}_{\beta \beta} & \mathbf{L}_{\beta \lambda} & \mathbf{L}_{\beta \theta} \\
\cdot & \cdot & \mathbf{L}_{\lambda \lambda} & \mathbf{L}_{\lambda \theta} \\
\cdot & \cdot & \cdot & \mathbf{L}_{\theta \theta}
\end{array}\right),
$$

whose elements are given in Appendix B. 
Under conditions that are fulfilled for parameters in the interior of the parameter space but not on the boundary, the asymptotic distribution of $(\widehat{\Theta}-\Theta)$ is $N_{4}\left(\mathbf{0}, I(\Theta)^{-1}\right)$, where $I(\Theta)$ is the expected information matrix. The multivariate normal $N_{4}\left(\mathbf{0}, J(\widehat{\Theta})^{-1}\right)$ distribution, where $I(\Theta)$ is replaced by $J(\widehat{\Theta})$, i.e., the observed information matrix evaluated at $\widehat{\Theta}$, can be used to construct approximate confidence intervals for the individual parameters.

We can compute the maximum values of the unrestricted and restricted log-likelihoods to obtain likelihood ratio (LF) statistics for testing some special models of the proposed family. Tests of the hypotheses of the type $H_{0}: \psi=\psi_{0}$ versus $H$ : $\psi \neq \psi_{0}$, where $\psi$ is a subset of parameters of $\Theta$, can be performed through LF statistics in the usual way.

\section{Simulation study}

\subsection{The Maximum Likelihood Estimator}

In this section, the Maximum likelihood estimators of parameters in density function 10 has been assessed by simulating for $(\theta, \lambda, \alpha, \beta)=(1,2,0.5,2)$.

The density function has been indicated in Figure 3. One can see three different states of ZBOLL-GHN density function.

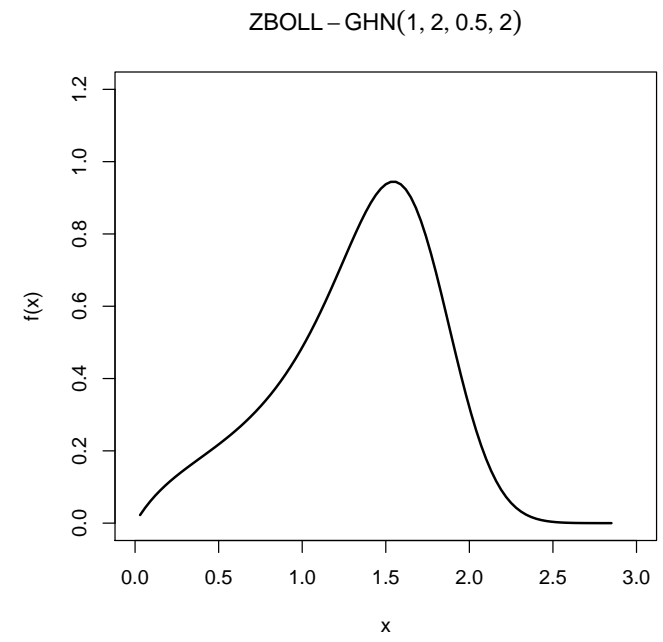

Figure 3. The density functions for simulation study.

To verify the validity of the maximum likelihood estimator, the Bias of MALE and the Mean Square Error of MLE (MSE) have been used. For example for $(\theta, \lambda, \alpha, \beta)=(1,2,0.5,2), r=1000$ times have been simulated samples of $n=20,21, \cdots, 110$. To estimate the numerical value of the maximum likelihood, the optim function (in the stat package) and Nelder-Mead method in R software has been used. If $\boldsymbol{\theta}=(\theta, \lambda, \alpha, \beta)$, for any simulation by $n$ volume and $i=1,2, \cdots, r$, the maximum likelihood estimates are obtained as $\widehat{\boldsymbol{\theta}}_{i}=(\hat{\theta}, \hat{\lambda}, \hat{\alpha}, \hat{\beta})$. To examine the performance of the MLE's for the ZBOLL-GHN distribution, we perform a simulation study:

1. Generate $r$ samples of size $n$ from equation (10).

2. Compute the MLE's for the $r$ samples, say $(\hat{\theta}, \hat{\lambda}, \hat{\alpha}, \hat{\beta})$ for $i=1,2, \ldots, r$.

3. Compute the standard errors of the MLE's for $r$ samples, say $\left(s_{\hat{\theta}}, s_{\hat{\lambda}}, s_{\hat{\alpha}}, s_{\hat{\beta}}\right)$ for $i=1,2, \ldots, r$.

4. Compute the MLE's, biases and mean squared errors given by

$$
\begin{gathered}
\operatorname{Bias}_{\widehat{\boldsymbol{\theta}}}(n)=\frac{1}{r} \sum_{i=1}^{r}\left(\widehat{\boldsymbol{\theta}}_{i}-\boldsymbol{\theta}_{i}\right), \\
\operatorname{MSE}_{\widehat{\boldsymbol{\theta}}}(n)=\frac{1}{r} \sum_{i=1}^{r}\left(\widehat{\boldsymbol{\theta}}_{i}-\boldsymbol{\theta}_{i}\right)^{2} .
\end{gathered}
$$


for $\boldsymbol{\theta}=\theta, \lambda, \alpha, \beta$.

We repeat these steps for $r=1000$ and $n=5,6, \ldots n^{*}\left(n^{*}\right.$ is different in each issue) with different values of $(\alpha, \beta, \lambda, \sigma)$, so computing $\operatorname{Bias}_{\widehat{\boldsymbol{\theta}}}(n)$ and $M S E_{\widehat{\boldsymbol{\theta}}}(n)$.

Figure 4, 5 respectively reveals how the four biases, mean squared errors vary with respect to $n$. As expected, the Biases and MSE's of estimated parameters converges to zero while $n$ growing.
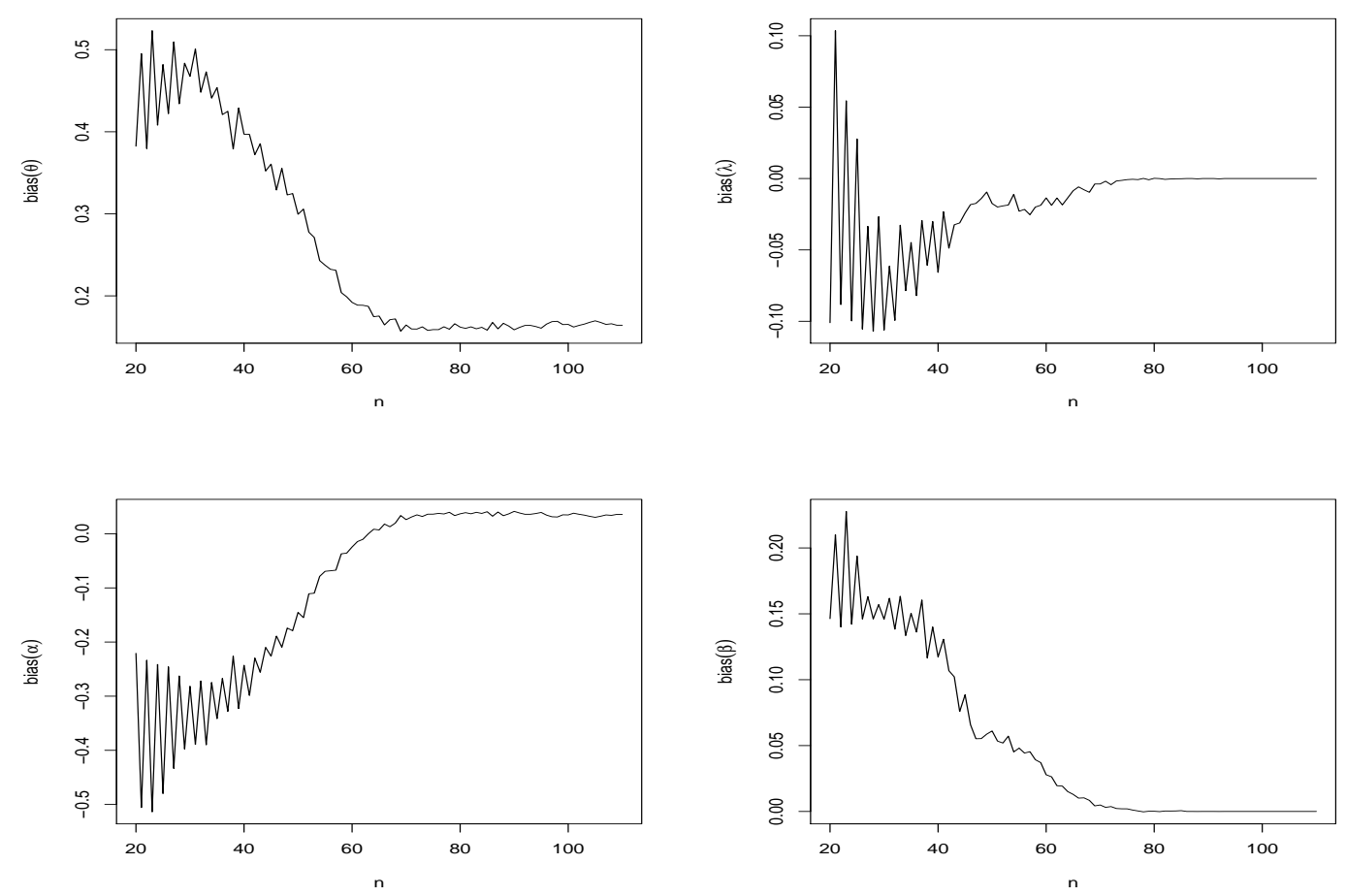

Figure 4. Bias of $\hat{\theta}, \hat{\lambda}, \hat{\alpha}, \hat{\beta}$ versus $n$ when $(\theta, \lambda, \alpha, \beta)=(1,2,0.5,2)$.

\subsection{The other estimation methods}

There are several approaches to estimate the parameters of distributions that each of them has its characteristic features and benefits. In this subsection five of those methods are briefly introduced and will be numerically investigated in the simulation study when $(\theta, \lambda, \alpha, \beta)=(1,2,0.5,2)$. A useful summary of these methods can be seen in Dey et al. [13]. Here $\left\{t_{i} ; i=1,2, \ldots, n\right\}$ is the associated order statistics and $F$ is the distribution function of ZBOLL-GHN.

6.2.1. Least squares and weighted least squares estimators The Least Squares (LSE) and weighted Least Squares Estimators (WLSE) are introduced by Swain et al. [26]. The LSE's and WLSE's are obtained by minimizing the following functions:

$$
\begin{gathered}
S_{\mathrm{LSE}}(\alpha, \beta, \theta, \lambda)=\sum_{i=1}^{n}\left(F\left(t_{i} ; \alpha, \beta\right)-\frac{i}{n+1}\right)^{2} \\
S_{\mathrm{WLSE}}(\alpha, \beta, \theta, \lambda)=\sum_{i=1}^{n} \frac{(n+1)^{2}(n+2)}{i(n-i+1)}\left(F\left(t_{i} ; \alpha, \beta\right)-\frac{i}{n+1}\right)^{2}
\end{gathered}
$$



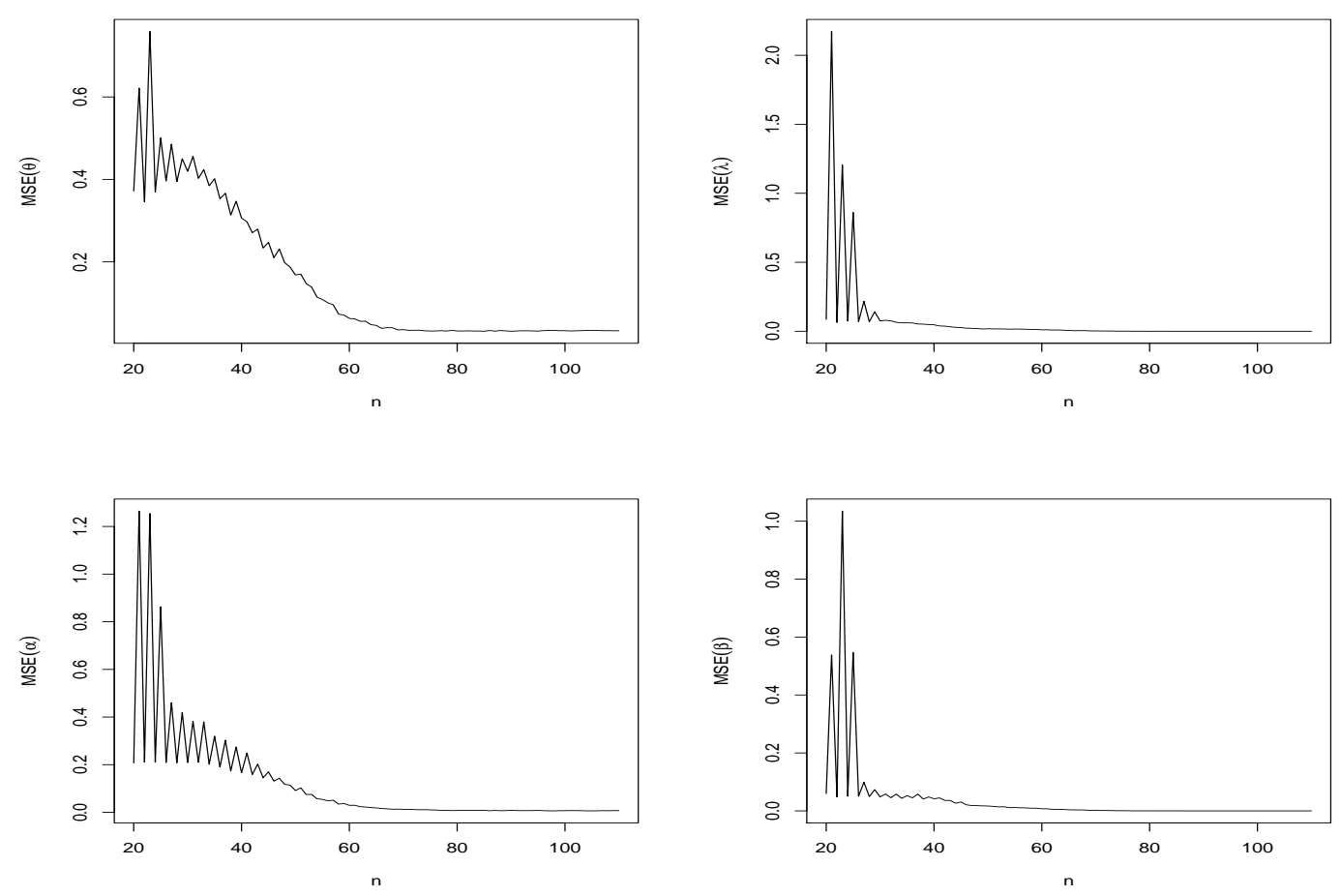

Figure 5. MSE of $\hat{\theta}, \hat{\lambda}, \hat{\alpha}, \hat{\beta}$ versus $n$ when $(\theta, \lambda, \alpha, \beta)=(1,2,0.5,2)$.

6.2.2. Cramér-von-Misers estimator Cramér-von-Misers Estimator (CME) is introduced by Choi and Bulgren [5]. The CME's is obtained by minimizing the following function:

$$
S_{\mathrm{ACME}}(\alpha, \beta, \theta, \lambda)=\frac{1}{12 n}+\sum_{i=1}^{n}\left(F\left(t_{i} ; \alpha, \beta\right)-\frac{2 i-1}{2 n}\right)^{2}
$$

6.2.3. Anderson-Darling and right-tailed Anderson-Darling The Anderson-Darling (ADE) and Right-Tailed Anderson-Darling Estimators (RTADE) are introduced by Anderson and Darling [4]. The ADE's and RTADE's are obtained by minimizing the following functions:

$$
\begin{aligned}
& S_{\mathrm{ADEN}}(\alpha, \beta, \theta, \lambda)=-n-\frac{1}{n} \sum_{i=1}^{n}(2 i-1)\left\{\log F\left(t_{i} ; \alpha, \beta\right)+\log \bar{F}\left(t_{n+1-i} ; \alpha, \beta\right)\right\} \\
& S_{\mathrm{TRADE}}(\alpha, \beta, \theta, \lambda)=\frac{n}{2}-2 \sum_{i=1}^{n} F\left(t_{i} ; \alpha, \beta\right)-\frac{1}{n} \sum_{i=1}^{n}(2 i-1) \log \bar{F}\left(t_{n+1-i} ; \alpha, \beta\right)
\end{aligned}
$$

where $\bar{F}(\cdot)=1-F(\cdot)$.

In order to explore the introduced above estimators we consider the one model that have been used in the subsection6.1, and we investigate MSEC of those estimators for different samples. For instance according to what has been mentioned in above, for $(\theta, \lambda, \alpha, \beta)=(1,2,0.5,2)$. we have simulated $\mathrm{r}=1000$ times with sample size of the $n=50,55,60, \cdots 550$ and then the MSE formula that are mentioned in the subsection 6.1 are calculated for them. To obtain the value of the estimators, we have used the optima function and Elder-Mead method in R.

The result of the simulations of this subsection is shown in figure 6. As it is clear from the MSE plot for two parameters with the increase in the volume of the sample all methods will approach to zero and this verifies the validity of the these estimation methods and numerical calculations for the distribution parameters ZBOLL-GHN. 

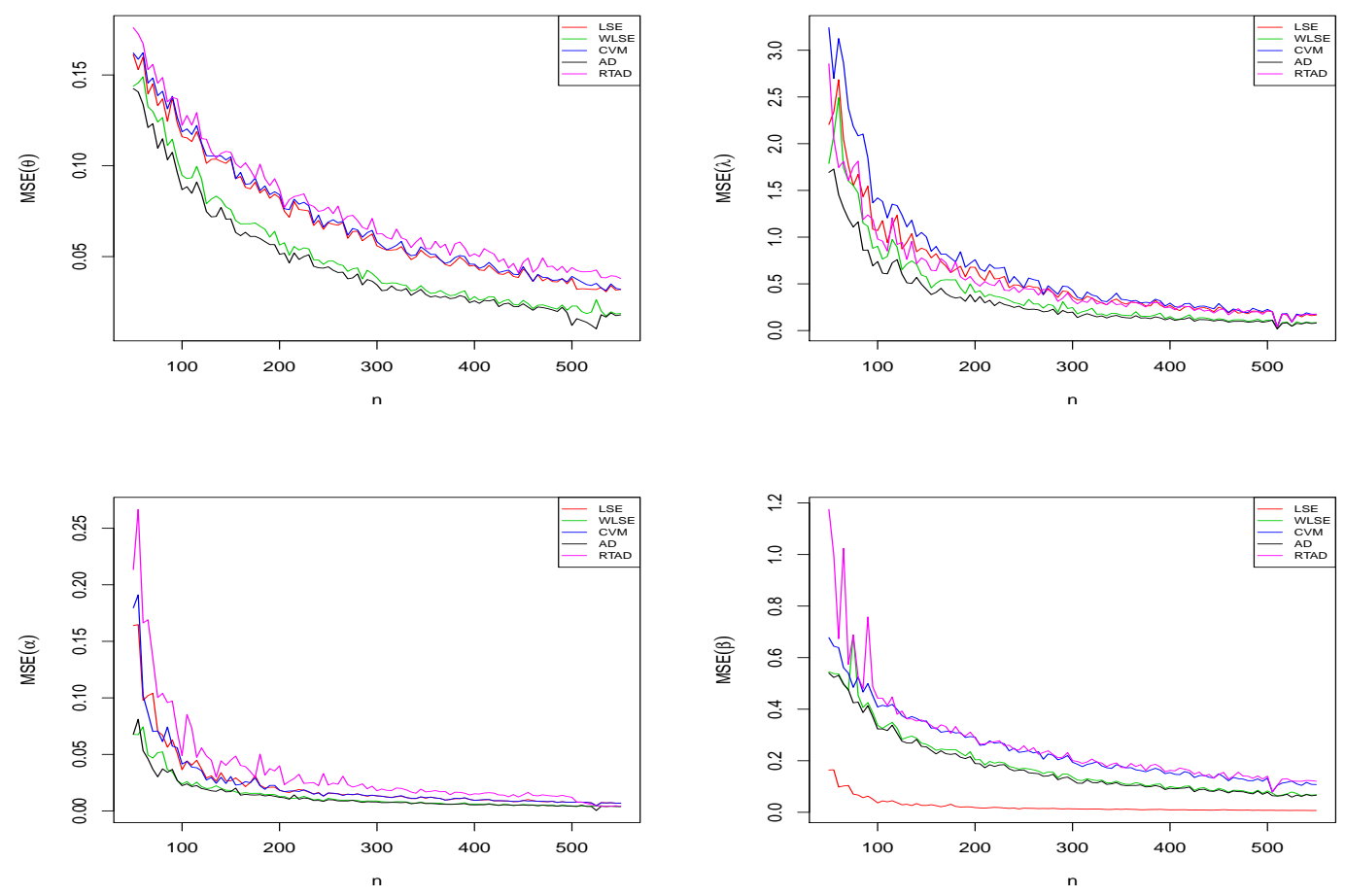

Figure 6. Bias and MSE of $\hat{\theta}, \hat{\lambda}, \hat{\alpha}, \hat{\beta}$ versus $n$ when $(\theta, \lambda, \alpha, \beta)=(1,2,0.5,2)$.

\section{Application}

In this section, fitting of (ZBOLL-GHN) family and some famous models to the a real data sets has been assessed. The Akaike information criterion (AIC), Anderson-Darling $\left(A^{*}\right)$ and Cramér-von Mises $\left(W^{*}\right)$, Kolmogorov-Smirnov (K.S) and the P-Value of K.S test, have been chosen to comparison of the models.

The distributions: Generalized Half Normal(GHN) (Cooray and Ananda [6]), Gamma Generalized Half Normal (GaGHN) ( Nadarajah ,et al. [22]), Odd Log Logistic Generalized Half Normal (OLL-GHN) ( Cordeiro, et al. [12]), Kumaraswamy-Generalized Half normal (KW-GHN) ( Cordeiro, et al. [8]), Beta- Generalized Half Normal (B-GHN) (Pescim et al. [23]), have been selected for comparison. The parameters of models have been estimated by the MLE method by using $R$ software.

\subsection{The strengths data}

This sub-section is related to study of data set which presented by Smith and Naylor [25] that include 63 observations. The data set is the strengths of $1.5 \mathrm{~cm}$ glass fibres, measured at the National Physical Laboratory, England. Unfortunately, the measurement units are not given in the article. The data set is the following:

$0.55,0.93,1.25,1.36,1.49,1.52,1.58,1.61,1.64,1.68,1.73,1.81,2,0.74,1.04,1.27,1.39,1.49,1.53,1.59,1.61,1.66$, $1.68,1.76,1.82,2.01,0.77,1.11,1.28,1.42,1.5,1.54,1.6,1.62,1.66,1.69,1.76,1.84,2.24,0.81,1.13,1.29,1.48,1.5,1.55$, $1.61,1.62,1.66,1.7,1.77,1.84,0.84,1.24,1.3,1.48,1.51,1.55,1.61,1.63,1.67,1.7,1.78,1.89$

\subsection{The survival times data}

The second data analyzed by Kundu et al. [21] and Cordeiro, et al. [8] correspond to 72 survival times of guinea pigs injected with different doses of tubercle bacilli. This data was analyzed by Cordeiro et al. [8] The data set is the following: 
Table 1. MLE's (standard errors in parentheses) for data.

\begin{tabular}{c|cccc}
\hline Model & \multicolumn{5}{|c}{ Estimates } \\
\hline ZBOLL-GHN $(\theta, \lambda, \alpha, \beta)$ & 2.2415 & 1.7182 & 5.8412 & 0.3399 \\
& $(0.6605)$ & $(2.0447)$ & $(7.5392)$ & $(0.1381)$ \\
GHN $(\theta, \lambda)$ & 1.6815 & 4.4145 & & \\
& $(0.0368)$ & $(0.4290)$ & & \\
OLL-GHN $(\theta, \lambda, \alpha)$ & 1.7086 & 3.7614 & 1.2903 & \\
& $(0.0480)$ & $(0.7746)$ & $(0.3282)$ & \\
Ga-GHN $(\theta, \lambda, \beta)$ & 1.5796 & 3.6699 & 1.3170 & \\
& $(0.1722)$ & $(1.0962)$ & $(0.5445)$ & \\
KW-GHN $(\theta, \lambda, a, b)$ & 1.2860 & 3.7835 & 1.0251 & 0.2247 \\
& $(0.0027)$ & $(0.0027)$ & $(0.2707)$ & $(0.0308)$ \\
B-GHN $(\theta, \lambda, a, b)$ & 1.3270 & 3.7761 & 1.0251 & 0.2247 \\
& $(0.0994)$ & $(0.1220)$ & $(0.2699)$ & $(0.1519)$ \\
\hline
\end{tabular}

Table 2. The $W^{*}, A^{*}$, AIC, CAIC, BIC, K-S and P-value statistics for data.

\begin{tabular}{c|ccccccc}
\hline Model & $W^{*}$ & $A^{*}$ & AIC & CAIC & BIC & K-S & P-value \\
\hline ZBOLL-GHN $(\theta, \lambda, \alpha, \beta)$ & 0.0943 & 0.5292 & 31.2544 & 31.9440 & 39.8269 & 0.1152 & 0.3732 \\
GHN $(\theta, \lambda)$ & 0.1873 & 1.0521 & 33.4807 & 33.6807 & 37.7670 & 0.1451 & 0.1410 \\
OLL-GHN $(\theta, \lambda, \alpha)$ & 0.1927 & 1.0650 & 34.3277 & 34.7345 & 40.7571 & 0.1368 & 0.1888 \\
Ga-GHN $(\theta, \lambda, \beta)$ & 0.1951 & 1.0845 & 35.0261 & 35.4329 & 41.4555 & 0.1444 & 0.1443 \\
KW-GHN $(\theta, \lambda, \mathrm{a}, \mathrm{b})$ & 0.1573 & 0.8917 & 35.2782 & 35.9679 & 43.8508 & 0.1307 & 0.2318 \\
B-GHN $(\theta, \lambda, \mathrm{a}, \mathrm{b})$ & 0.1637 & 0.9222 & 36.0809 & 36.7706 & 44.6534 & 0.1265 & 0.2655 \\
\hline
\end{tabular}

$12,15,22,24,24,32,32,33,34,38,38,43,44,48,52,53,54,54,55,56,57,58,58,59,60,60,60,60,61,62,63,65$, $65,67,68,70,70,72,73,75,76,76,81,83,84,85,87,91,95,96,98,99,109,110,121,127,129,131,143,146,146,175$, $175,211,233,258,258,263,297,341,341,376$.

Table 3. MLE's (standard errors in parentheses) for data.

\begin{tabular}{c|cccc}
\hline Model & \multicolumn{5}{|c}{ Estimates } \\
\hline ZBOLL-GHN $(\theta, \lambda, \alpha, \beta)$ & 280.5393 & 0.2207 & 5.8836 & 1.6816 \\
& $(910.2018)$ & $(0.3392)$ & $(10.2114)$ & $(0.9849)$ \\
B-GHN $(\theta, \lambda, a, b)$ & 127.0368 & 0.1419 & 61.7598 & 33.4539 \\
& $(564.1019)$ & $(0.1004)$ & $(89.4203)$ & $(74.3531)$ \\
OLL-GHN $(\theta, \lambda, \alpha)$ & 179.2866 & 0.4569 & 3.0677 & \\
& $(38.9724)$ & $(0.1187)$ & $(0.9159)$ & \\
KW-GHN $(\theta, \lambda, a, b)$ & 134.8116 & 0.2557 & 7.4712 & 23.3122 \\
& $(6.5539)$ & $(0.1883)$ & $(6.9646)$ & $(29.8037)$ \\
$\operatorname{GHN}(\theta, \lambda)$ & 129.2614 & 1.0164 & & \\
& $(11.8996)$ & $(0.0911)$ & & \\
$\operatorname{Ga}-\operatorname{GHN}(\theta, \lambda, \beta)$ & 150.4775 & 1.1050 & 0.9122 & \\
& $(169.1728)$ & $(0.9206)$ & $(1.1471)$ \\
\hline
\end{tabular}

In the Tables 1-4, a summary of the fitted information criteria and estimated MLEs for two data sets with different models have come, respectively. Models have been sorted from the lowest to the highest value of AIC. As you see, the (ZBOLL$\mathrm{GHN}$ ) is selected as the best model with more criteria. Note that p-value for (ZBOLL-GHN) is also more than all other 


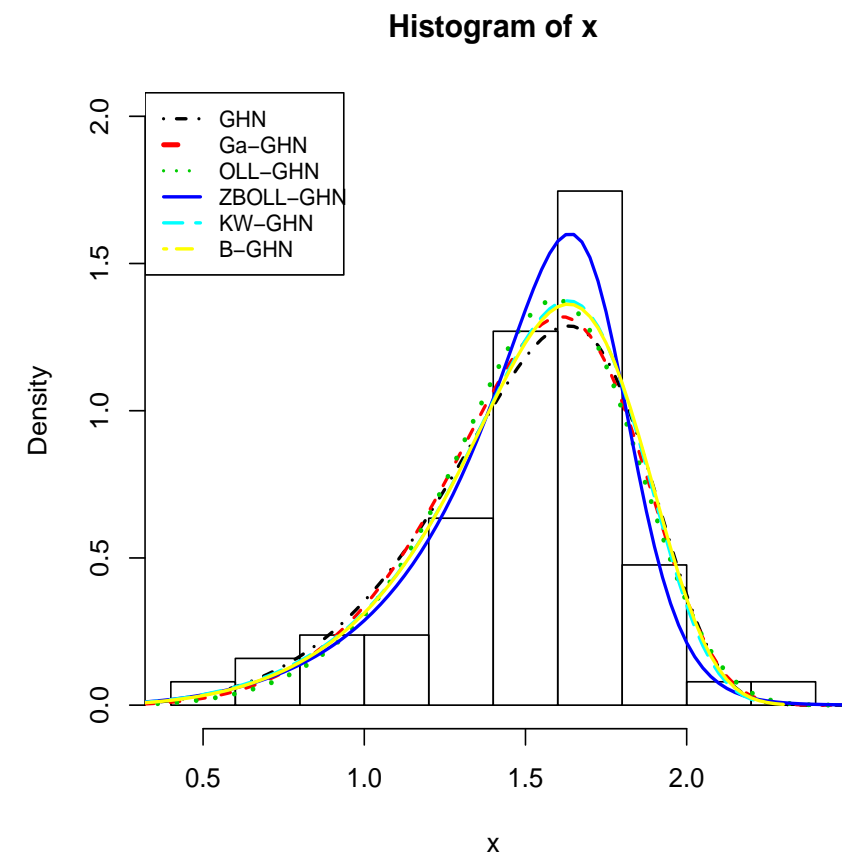

Figure 7. Fitted GHN, Ga-GHN, OLL-GHN, ZBOLL-GHN, KW-GHN, and B-GHN densities for data.

Table 4. The $W^{*}, A^{*}$, AIC, CAIC, BIC, K-S and P-value statistics for data.

\begin{tabular}{c|ccccccc}
\hline Model & $W^{*}$ & $A^{*}$ & AIC & CAIC & BIC & K-S & P-value \\
\hline ZOLL-GHN $(\theta, \lambda, \alpha, \beta)$ & 0.1060 & 0.5951 & 787.1191 & 787.7162 & 3796.2258 & 0.0886 & 0.6243 \\
B-GHN $(\theta, \lambda, \mathrm{a}, \mathrm{b})$ & 0.1870 & 1.0096 & 789.5217 & 789.6284 & 790.1187 & 0.1014 & 0.4497 \\
LOL-GHN $(\theta, \lambda, \alpha)$ & 0.2239 & 1.2336 & 790.3319 & 790.6849 & 797.1619 & 0.1019 & 0.4439 \\
KW-GHN $(\theta, \lambda, \mathrm{a}, \mathrm{b})$ & 0.3441 & 1.8876 & 797.1904 & 797.7874 & 806.2971 & 0.1317 & 0.1644 \\
GHN $(\theta, \lambda)$ & 0.5801 & 3.1970 & 807.4749 & 807.6488 & 812.0282 & 0.58011 & 3.1970 \\
Ga-GHN $(\theta, \lambda, \beta)$ & 0.5919 & 3.2589 & 811.4317 & 811.7846 & 818.2617 & 0.2072 & 0.0041 \\
\hline
\end{tabular}

distributions. The histogram of the data sets and the plots of fitted pdf are displayed in fig 7 and fig 8 in two parts, including special case and other pdfs.

\section{Conclusions}

In this paper, we introduce a new Zografos-Balakrishnan odd log- logistic generalized half-normal ZBOLL-GHN family of distributions. Some of it's properties are derived. Different methods have been used to estimate it's parameters. The maximum likelihood estimators are assessed with simulated real data from proposed model. We conclude from the Bias and MSE plots with the increase in the volume of the sample, all methods will approach to zero and this verifies the validity of the these estimation methods. The ZBOLL-GHN is applied to fit two real data sets. The flexibility of this distribution is assessed by applying it to real data sets and comparing it with other distributions. These applications show that it has the ability to fit skewed (left or right) and heavy-tailed data due to its flexibility. The results of tables and figures illustrate the new models provide consistently better fits than other competitive models for these data sets. 


\section{Histogram of $x$}

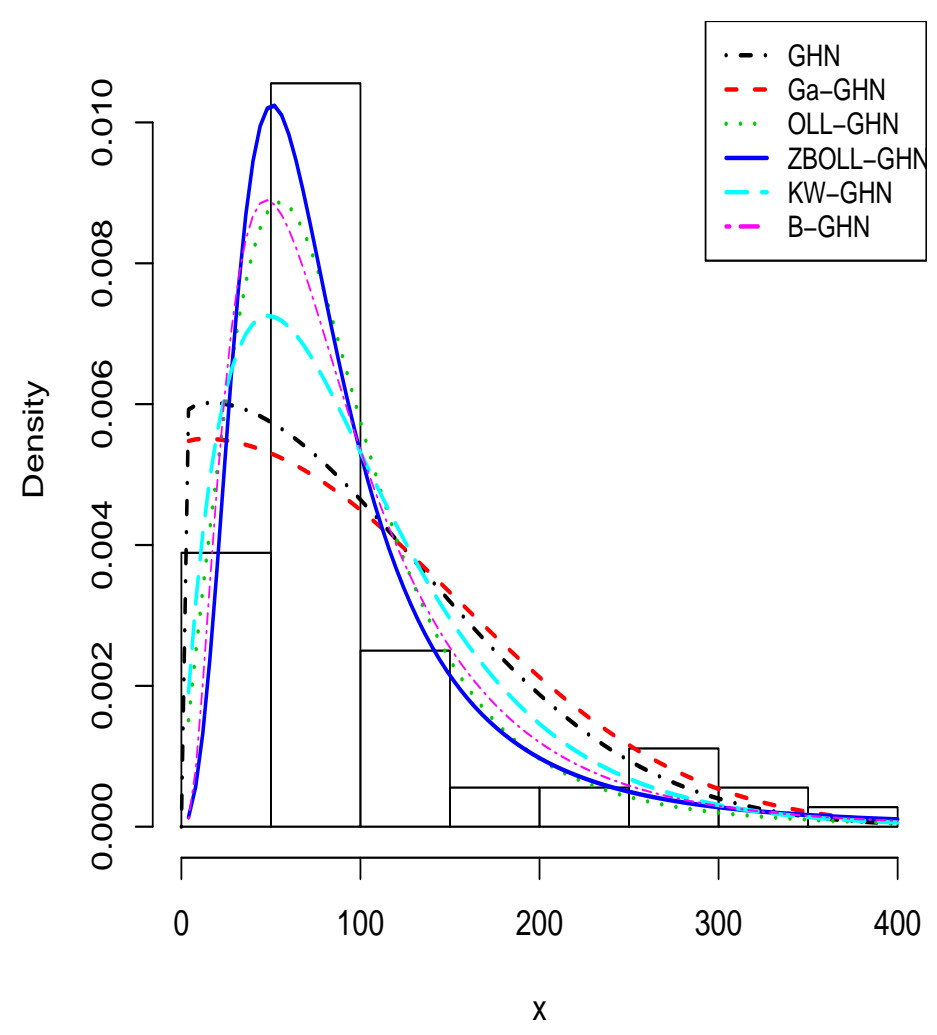

Figure 8. Fitted GHN, Ga-GHN, OLL-GHN, ZBOLL-GHN, KW-GHN and B-GHN densities for data.

\section{Appendix A: Three useful power series}

We present three power series required for the algebraic developments in Sections 3 and 4.2. First, for $b>0$ real non-integer and $-1<u<1$, we have the binomial expansion

$$
(1-u)^{a}=\sum_{j=0}^{\infty}(-1)^{j}\left(\begin{array}{l}
a \\
j
\end{array}\right) u^{j},
$$

where the binomial coefficient is defined for any real.

Second, expanding $z^{\lambda}$ in Taylor series, we can write

$$
z^{\lambda}=\sum_{k=0}^{\infty}(\lambda)_{k}(z-1)^{k} / k !=\sum_{i=0}^{\infty} f_{i} z^{i}
$$

where

$$
f_{i}=f_{i}(\lambda)=\sum_{k=i}^{\infty} \frac{(-1)^{k-i}}{k !}\left(\begin{array}{c}
k \\
i
\end{array}\right)(\lambda)_{k}
$$

and $(\lambda)_{k}=\lambda(\lambda-1) \ldots(\lambda-k+1)$ denotes the descending factorial. 
Third, we obtain an expansion for $\left[G(x)^{a}+\bar{G}(x)^{a}\right]^{c}$. We can write from equation (30) and (29)

$$
\left[G(x)^{a}+\bar{G}(x)^{a}\right]=\sum_{j=0}^{\infty} t_{j} G(x)^{j},
$$

where $t_{j}=t_{j}(a)=a_{j}(a)+(-1)^{j}\left(\begin{array}{l}a \\ j\end{array}\right)$ and $a_{j}(a)$ is defined by (30). Then, using (30), we have

$$
\left[G(x)^{a}+\bar{G}(x)^{a}\right]^{c}=\sum_{i=0}^{\infty} f_{i}\left(\sum_{j=0}^{\infty} t_{j} G(x)^{j}\right)^{i},
$$

where $f_{i}=f_{i}(c)$.

Finally, using again equations (31) and (32), we have

$$
\left[G(x)^{a}+\bar{G}(x)^{a}\right]^{c}=\sum_{j=0}^{\infty} h_{j}(a, c) G(x)^{j},
$$

where $h_{j}(a, c)=\sum_{i=0}^{\infty} f_{i} m_{i, j}$ and (for $\left.i \geq 0\right) m_{i, j}=\left(j t_{0}\right)^{-1} \sum_{m=1}^{j}[m(j+1)-j] t_{m} m_{i, j-m}$ (for $\left.j \geq 1\right)$ and $m_{i, 0}=$ $t_{0}^{i}$.

\section{Appendix B}

The elements of the observed information matrix $J(\theta)$ for the parameters $(\alpha, \beta, \boldsymbol{\tau})$ are given by the following:

$$
\begin{aligned}
& J_{\alpha \alpha}=\frac{-n}{\alpha^{2}}-2 \sum_{i=1}^{n} \frac{\left(q_{i}-1\right)^{\alpha}\left[\log \left(q_{i}-1\right)\right]^{2}+\left(2-q_{i}\right)^{\alpha}\left[\log \left(2-q_{i}\right)\right]^{2}}{\left(q_{i}-1\right)^{\alpha}+\left(2-q_{i}\right)^{\alpha}} \\
&+2 \sum_{i=1}^{n}\left[\frac{\left(q_{i}-1\right)^{\alpha} \log \left(q_{i}-1\right)+\left(2-q_{i}\right)^{\alpha} \log \left(2-q_{i}\right)}{\left(q_{i}-1\right)^{\alpha}+\left(2-q_{i}\right)^{\alpha}}\right]^{2} \\
&+(\beta-1) \sum_{i=1}^{n} \frac{\left(q_{i}-1\right)^{\alpha} \log \left(q_{i}-1\right) \log \left(\frac{q_{i}-1}{2-q_{i}}\right)}{\left[\left(q_{i}-1\right)^{\alpha}+\left(2-q_{i}\right)^{\alpha}\right] \log \left\{\frac{\left(2-q_{i}\right)^{\alpha}}{\left(q_{i}-1\right)^{\alpha}+\left(2-q_{i}\right)^{\alpha}}\right\}} \\
&+(\beta-1) \sum_{i=1}^{n} \frac{\left(q_{i}-1\right)^{\alpha} \log \left(\frac{q_{i}-1}{2-q_{i}}\right)\left[\left(q_{i}-1\right)^{\alpha} \log \left(q_{i}-1\right)+\left(2-q_{i}\right)^{\alpha} \log \left(2-q_{i}\right)\right]}{\left[\left(q_{i}-1\right)^{\alpha}+\left(2-q_{i}\right)^{\alpha}\right]^{2} \log \left\{\frac{\left(2-q_{i}\right)^{\alpha}}{\left(q_{i}-1\right)^{\alpha}+\left(2-q_{i}\right)^{\alpha}}\right\}} \\
&+(\beta-1) \sum_{i=1}^{n} \frac{\left(q_{i}-1\right)^{\alpha} \log \left(\frac{q_{i}-1}{2-q_{i}}\right)\left[\left(q_{i}-1\right)^{\alpha} \log \left(q_{i}-1\right)+\left(2-q_{i}\right)^{\alpha} \log \left(2-q_{i}\right)\right]}{\left.\left\{\left[q_{i}-1\right)^{\alpha}+\left(2-q_{i}\right)^{\alpha}\right] \log \left\{\frac{\left(2-q_{i}\right)^{\alpha}}{\left(q_{i}-1\right)^{\alpha}+\left(2-q_{i}\right)^{\alpha}}\right\}\right\}^{2}} \\
&-(\beta-1) \sum_{i=1}^{n} \frac{\left(q_{i}-1\right)^{\alpha} \log \left(2-q_{i}\right) \log \left(\frac{q_{i}-1}{2-q_{i}}\right)}{\left[\left(q_{i}-1\right)^{\alpha}+\left(2-q_{i}\right)^{\alpha}\right]\left\{\log \left\{\frac{\left(2-q_{i}\right)^{\alpha}}{\left(q_{i}-1\right)^{\alpha}+\left(2-q_{i}\right)^{\alpha}}\right\}\right\}^{2}}, \\
& J_{\alpha \beta}=\sum_{i=1}^{n} \frac{\left(q_{i}-1\right)^{\alpha} \log \left(\frac{q_{i}-1}{2-q_{i}}\right)}{\left[\left(q_{i}-1\right)^{\alpha}+\left(2-q_{i}\right)^{\alpha}\right] \log \left\{\frac{\left(2-q_{i}\right)^{\alpha}}{\left(q_{i}-1\right)^{\alpha}+\left(2-q_{i}\right)^{\alpha}}\right\}},
\end{aligned}
$$




$$
\begin{aligned}
& J_{\alpha \lambda}=\sum_{i=1}^{n} \frac{q_{i}^{(\lambda)}}{q_{i}-1}-\sum_{i=1}^{n} \frac{q_{i}^{(\lambda)}}{2-q_{i}}-2 \sum_{i=1}^{n} q_{i}^{(\lambda)} \frac{\left(q_{i}-1\right)^{\alpha-1}-\left(2-q_{i}\right)^{\alpha-1}}{\left(q_{i}-1\right)^{\alpha}+\left(2-q_{i}\right)^{\alpha}} \\
& -2 \alpha \sum_{i=1}^{n} q_{i}^{(\lambda)} \frac{\left(q_{i}-1\right)^{\alpha-1} \log \left(q_{i}-1\right)-\left(2-q_{i}\right)^{\alpha-1} \log \left(2-q_{i}\right)}{\left(q_{i}-1\right)^{\alpha}+\left(2-q_{i}\right)^{\alpha}} \\
& +2 \alpha \sum_{i=1}^{n} q_{i}^{(\lambda)} \frac{\left[\left(q_{i}-1\right)^{\alpha-1}-\left(2-q_{i}\right)^{\alpha-1}\right]\left[\left(q_{i}-1\right)^{\alpha} \log \left(q_{i}-1\right)+\left(2-q_{i}\right)^{\alpha} \log \left(2-q_{i}\right)\right]}{\left[\left(q_{i}-1\right)^{\alpha}+\left(2-q_{i}\right)^{\alpha}\right]^{2}} \\
& +\alpha(\beta-1) \sum_{i=1}^{n} q_{i}^{(\lambda)} \frac{\left(q_{i}-1\right)^{\alpha} \log \left(\frac{q_{i}-1}{2-q_{i}}\right)}{\left[\left(q_{i}-1\right)^{\alpha}+\left(2-q_{i}\right)^{\alpha}\right] \log \left\{\frac{\left(2-q_{i}\right)^{\alpha}}{\left(q_{i}-1\right)^{\alpha}+\left(2-q_{i}\right)^{\alpha}}\right\}} \\
& +(\beta-1) \sum_{i=1}^{n} q_{i}^{(\lambda)} \frac{\left(q_{i}-1\right)^{\alpha-1}\left(2-q_{i}\right)^{-1}}{\left[\left(q_{i}-1\right)^{\alpha}+\left(2-q_{i}\right)^{\alpha}\right] \log \left\{\frac{\left(2-q_{i}\right)^{\alpha}}{\left(q_{i}-1\right)^{\alpha}+\left(2-q_{i}\right)^{\alpha}}\right\}} \\
& -\alpha(\beta-1) \sum_{i=1}^{n} q_{i}^{(\lambda)} \frac{\left(q_{i}-1\right)^{\alpha}\left[\left(q_{i}-1\right)^{\alpha-1}+\left(2-q_{i}\right)^{\alpha-1}\right] \log \left(\frac{q_{i}-1}{2-q_{i}}\right)}{\left[\left(q_{i}-1\right)^{\alpha}+\left(2-q_{i}\right)^{\alpha}\right]^{2} \log \left\{\frac{\left(2-q_{i}\right)^{\alpha}}{\left(q_{i}-1\right)^{\alpha}+\left(2-q_{i}\right)^{\alpha}}\right\}} \\
& +\alpha(\beta-1) \sum_{i=1}^{n} q_{i}^{(\lambda)} \frac{\left(q_{i}-1\right)^{\alpha}\left(2-q_{i}\right)^{-1} \log \left(\frac{q_{i}-1}{2-q_{i}}\right)}{\left\{\log \left\{\frac{\left(2-q_{i}\right)^{\alpha}}{\left(q_{i}-1\right)^{\alpha}+\left(2-q_{i}\right)^{\alpha}}\right\}\right\}^{2}} \\
& +\alpha(\beta-1) \sum_{i=1}^{n} q_{i}^{(\lambda)} \frac{\left(q_{i}-1\right)^{\alpha}\left[\left(q_{i}-1\right)^{\alpha-1}-\left(2-q_{i}\right)^{\alpha-1}\right] \log \left(\frac{q_{i}-1}{2-q_{i}}\right)}{\left[\left(q_{i}-1\right)^{\alpha}+\left(2-q_{i}\right)^{\alpha}\right]\left\{\log \left\{\frac{\left(2-q_{i}\right)^{\alpha}}{\left(q_{i}-1\right)^{\alpha}+\left(2-q_{i}\right)^{\alpha}}\right\}\right\}^{2}}, \\
& J_{\alpha \theta}=\sum_{i=1}^{n} \frac{q_{i}^{(\theta)}}{q_{i}-1}-\sum_{i=1}^{n} \frac{q_{i}^{(\theta)}}{2-q_{i}}-2 \sum_{i=1}^{n} q_{i}^{(\theta)} \frac{\left(q_{i}-1\right)^{\alpha-1}-\left(2-q_{i}\right)^{\alpha-1}}{\left(q_{i}-1\right)^{\alpha}+\left(2-q_{i}\right)^{\alpha}} \\
& -2 \alpha \sum_{i=1}^{n} q_{i}^{(\theta)} \frac{\left(q_{i}-1\right)^{\alpha-1} \log \left(q_{i}-1\right)-\left(2-q_{i}\right)^{\alpha-1} \log \left(2-q_{i}\right)}{\left(q_{i}-1\right)^{\alpha}+\left(2-q_{i}\right)^{\alpha}} \\
& +2 \alpha \sum_{i=1}^{n} q_{i}^{(\theta)} \frac{\left[\left(q_{i}-1\right)^{\alpha-1}-\left(2-q_{i}\right)^{\alpha-1}\right]\left[\left(q_{i}-1\right)^{\alpha} \log \left(q_{i}-1\right)+\left(2-q_{i}\right)^{\alpha} \log \left(2-q_{i}\right)\right]}{\left[\left(q_{i}-1\right)^{\alpha}+\left(2-q_{i}\right)^{\alpha}\right]^{2}} \\
& +\alpha(\beta-1) \sum_{i=1}^{n} q_{i}^{(\theta)} \frac{\left(q_{i}-1\right)^{\alpha} \log \left(\frac{q_{i}-1}{2-q_{i}}\right)}{\left[\left(q_{i}-1\right)^{\alpha}+\left(2-q_{i}\right)^{\alpha}\right] \log \left\{\frac{\left(2-q_{i}\right)^{\alpha}}{\left(q_{i}-1\right)^{\alpha}+\left(2-q_{i}\right)^{\alpha}}\right\}} \\
& +(\beta-1) \sum_{i=1}^{n} q_{i}^{(\theta)} \frac{\left(q_{i}-1\right)^{\alpha-1}\left(2-q_{i}\right)^{-1}}{\left[\left(q_{i}-1\right)^{\alpha}+\left(2-q_{i}\right)^{\alpha}\right] \log \left\{\frac{\left(2-q_{i}\right)^{\alpha}}{\left(q_{i}-1\right)^{\alpha}+\left(2-q_{i}\right)^{\alpha}}\right\}} \\
& -\alpha(\beta-1) \sum_{i=1}^{n} q_{i}^{(\theta)} \frac{\left(q_{i}-1\right)^{\alpha}\left[\left(q_{i}-1\right)^{\alpha-1}+\left(2-q_{i}\right)^{\alpha-1}\right] \log \left(\frac{q_{i}-1}{2-q_{i}}\right)}{\left[\left(q_{i}-1\right)^{\alpha}+\left(2-q_{i}\right)^{\alpha}\right]^{2} \log \left\{\frac{\left(2-q_{i}\right)^{\alpha}}{\left(q_{i}-1\right)^{\alpha}+\left(2-q_{i}\right)^{\alpha}}\right\}} \\
& +\alpha(\beta-1) \sum_{i=1}^{n} q_{i}^{(\theta)} \frac{\left(q_{i}-1\right)^{\alpha}\left(2-q_{i}\right)^{-1} \log \left(\frac{q_{i}-1}{2-q_{i}}\right)}{\left\{\log \left\{\frac{\left(2-q_{i}\right)^{\alpha}}{\left(q_{i}-1\right)^{\alpha}+\left(2-q_{i}\right)^{\alpha}}\right\}\right\}^{2}} \\
& +\alpha(\beta-1) \sum_{i=1}^{n} q_{i}^{(\theta)} \frac{\left(q_{i}-1\right)^{\alpha}\left[\left(q_{i}-1\right)^{\alpha-1}-\left(2-q_{i}\right)^{\alpha-1}\right] \log \left(\frac{q_{i}-1}{2-q_{i}}\right)}{\left[\left(q_{i}-1\right)^{\alpha}+\left(2-q_{i}\right)^{\alpha}\right]\left\{\log \left\{\frac{\left(2-q_{i}\right)^{\alpha}}{\left(q_{i}-1\right)^{\alpha}+\left(2-q_{i}\right)^{\alpha}}\right\}\right\}^{2}}
\end{aligned}
$$




$$
J_{\beta \beta}=-n \psi^{\prime}(\beta)
$$

$$
J_{\beta \lambda}=-\alpha \sum_{i=1}^{n} q_{i}^{(\lambda)} \frac{\left(q_{i}-1\right)^{\alpha-1}\left(2-q_{i}\right)^{-1}}{\left[\left(q_{i}-1\right)^{\alpha}+\left(2-q_{i}\right)^{\alpha}\right] \log \left\{\frac{\left(2-q_{i}\right)^{\alpha}}{\left(q_{i}-1\right)^{\alpha}+\left(2-q_{i}\right)^{\alpha}}\right\}},
$$

$$
J_{\beta \theta}=-\alpha \sum_{i=1}^{n} q_{i}^{(\theta)} \frac{\left(q_{i}-1\right)^{\alpha-1}\left(2-q_{i}\right)^{-1}}{\left[\left(q_{i}-1\right)^{\alpha}+\left(2-q_{i}\right)^{\alpha}\right] \log \left\{\frac{\left(2-q_{i}\right)^{\alpha}}{\left(q_{i}-1\right)^{\alpha}+\left(2-q_{i}\right)^{\alpha}}\right\}},
$$

$$
\begin{aligned}
& J_{\lambda \lambda}=-\frac{n}{\lambda^{2}}-2 \sum_{i=1}^{n}\left(\frac{x_{i}}{\theta}\right)^{2 \lambda}\left[\log \left(\frac{x_{i}}{\theta}\right)\right]^{2}+(\alpha-1) \sum_{i=1}^{n} \frac{q_{i}^{(\lambda \lambda)}\left(q_{i}-1\right)-\left[q_{i}^{(\lambda)}\right]^{2}}{\left(q_{i}-1\right)^{2}} \\
& -(\alpha-1) \sum_{i=1}^{n} \frac{q_{i}^{(\lambda \lambda)}\left(2-q_{i}\right)+\left[q_{i}^{(\lambda)}\right]^{2}}{\left(2-q_{i}\right)^{2}} \\
& -2 \alpha \sum_{i=1}^{n} q_{i}^{(\lambda \lambda)} \frac{\left(q_{i}-1\right)^{\alpha-1}-\left(2-q_{i}\right)^{\alpha-1}}{\left(q_{i}-1\right)^{\alpha}+\left(2-q_{i}\right)^{\alpha}} \\
& -2 \alpha(\alpha-1) \sum_{i=1}^{n}\left[q_{i}^{(\lambda)}\right]^{2} \frac{\left(q_{i}-1\right)^{\alpha-2}+\left(2-q_{i}\right)^{\alpha-2}}{\left(q_{i}-1\right)^{\alpha}+\left(2-q_{i}\right)^{\alpha}} \\
& +2 \alpha^{2} \sum_{i=1}^{n}\left[q_{i}^{(\lambda)} \frac{\left(q_{i}-1\right)^{\alpha-1}-\left(2-q_{i}\right)^{\alpha-1}}{\left(q_{i}-1\right)^{\alpha}+\left(2-q_{i}\right)^{\alpha}}\right]^{2} \\
& -\alpha(\beta-1) \sum_{i=1}^{n} \frac{\left(2-q_{i}\right)^{-1}\left(q_{i}-1\right)^{\alpha-2}\left[q_{i}^{(\lambda \lambda)}\left(q_{i}-1\right)+(\alpha-1)\left[q_{i}^{(\lambda)}\right]^{2}\right]}{\left[\left(q_{i}-1\right)^{\alpha}+\left(2-q_{i}\right)^{\alpha}\right] \log \left\{\frac{\left(2-q_{i}\right)^{\alpha}}{\left(q_{i}-1\right)^{\alpha}+\left(2-q_{i}\right)^{\alpha}}\right\}} \\
& +\alpha^{2}(\beta-1) \sum_{i=1}^{n}\left[q_{i}^{(\lambda)}\right]^{2} \frac{\left(2-q_{i}\right)^{-1}\left(q_{i}-1\right)^{\alpha-1}\left[\left(q_{i}-1\right)^{\alpha-1}-\left(2-q_{i}\right)^{\alpha-1}\right]}{\left[\left(q_{i}-1\right)^{\alpha}+\left(2-q_{i}\right)^{\alpha}\right]^{2} \log \left\{\frac{\left(2-q_{i}\right)^{\alpha}}{\left(q_{i}-1\right)^{\alpha}+\left(2-q_{i}\right)^{\alpha}}\right\}} \\
& -\alpha(\beta-1) \sum_{i=1}^{n}\left[q_{i}^{(\lambda)}\right]^{2} \frac{\left(2-q_{i}\right)^{-2}\left(q_{i}-1\right)^{\alpha-1}}{\left[\left(q_{i}-1\right)^{\alpha}+\left(2-q_{i}\right)^{\alpha}\right] \log \left\{\frac{\left(2-q_{i}\right)^{\alpha}}{\left(q_{i}-1\right)^{\alpha}+\left(2-q_{i}\right)^{\alpha}}\right\}} \\
& -\alpha^{2}(\beta-1) \sum_{i=1}^{n}\left\{q_{i}^{(\lambda)} \frac{\left(2-q_{i}\right)^{-1}\left(q_{i}-1\right)^{\alpha-1}}{\left[\left(q_{i}-1\right)^{\alpha}+\left(2-q_{i}\right)^{\alpha}\right] \log \left\{\frac{\left(2-q_{i}\right)^{\alpha}}{\left(q_{i}-1\right)^{\alpha}+\left(2-q_{i}\right)^{\alpha}}\right\}}\right\}^{2},
\end{aligned}
$$




$$
\begin{aligned}
& J_{\lambda \theta}=-\frac{n}{\theta}-2 \frac{\lambda}{\theta} \sum_{i=1}^{n}\left(\frac{x_{i}}{\theta}\right)^{2 \lambda} \log \left(\frac{x_{i}}{\theta}\right)+\frac{1}{\theta} \sum_{i=1}^{n}\left(\frac{x_{i}}{\theta}\right)^{2 \lambda} \\
& +(\alpha-1) \sum_{i=1}^{n} \frac{q_{i}^{(\lambda \theta)}\left(q_{i}-1\right)-q_{i}^{(\lambda)} q_{i}^{(\theta)}}{\left(q_{i}-1\right)^{2}} \\
& -\quad(\alpha-1) \sum_{i=1}^{n} \frac{q_{i}^{(\lambda \theta)}\left(2-q_{i}\right)+q_{i}^{(\lambda)} q_{i}^{(\theta)}}{\left(2-q_{i}\right)^{2}} \\
& -2 \alpha \sum_{i=1}^{n} q_{i}^{(\lambda \theta)} \frac{\left(q_{i}-1\right)^{\alpha-1}-\left(2-q_{i}\right)^{\alpha-1}}{\left(q_{i}-1\right)^{\alpha}+\left(2-q_{i}\right)^{\alpha}} \\
& -2 \alpha(\alpha-1) \sum_{i=1}^{n} q_{i}^{(\lambda)} q_{i}^{(\theta)} \frac{\left(q_{i}-1\right)^{\alpha-2}+\left(2-q_{i}\right)^{\alpha-2}}{\left(q_{i}-1\right)^{\alpha}+\left(2-q_{i}\right)^{\alpha}} \\
& +2 \alpha^{2} \sum_{i=1}^{n} q_{i}^{(\lambda)} q_{i}^{(\theta)}\left[\frac{\left(q_{i}-1\right)^{\alpha-1}-\left(2-q_{i}\right)^{\alpha-1}}{\left(q_{i}-1\right)^{\alpha}+\left(2-q_{i}\right)^{\alpha}}\right]^{2} \\
& -\alpha(\beta-1) \sum_{i=1}^{n} \frac{\left(2-q_{i}\right)^{-1}\left(q_{i}-1\right)^{\alpha-2}\left[q_{i}^{(\lambda \theta)}\left(q_{i}-1\right)+(\alpha-1) q_{i}^{(\lambda)} q_{i}^{(\theta)}\right]}{\left[\left(q_{i}-1\right)^{\alpha}+\left(2-q_{i}\right)^{\alpha}\right] \log \left\{\frac{\left(2-q_{i}\right)^{\alpha}}{\left(q_{i}-1\right)^{\alpha}+\left(2-q_{i}\right)^{\alpha}}\right\}} \\
& +\alpha^{2}(\beta-1) \sum_{i=1}^{n} q_{i}^{(\lambda)} q_{i}^{(\theta)} \frac{\left(2-q_{i}\right)^{-1}\left(q_{i}-1\right)^{\alpha-1}\left[\left(q_{i}-1\right)^{\alpha-1}-\left(2-q_{i}\right)^{\alpha-1}\right]}{\left[\left(q_{i}-1\right)^{\alpha}+\left(2-q_{i}\right)^{\alpha}\right]^{2} \log \left\{\frac{\left(2-q_{i}\right)^{\alpha}}{\left(q_{i}-1\right)^{\alpha}+\left(2-q_{i}\right)^{\alpha}}\right\}} \\
& -\alpha(\beta-1) \sum_{i=1}^{n} q_{i}^{(\lambda)} q_{i}^{(\theta)} \frac{\left(2-q_{i}\right)^{-2}\left(q_{i}-1\right)^{\alpha-1}}{\left[\left(q_{i}-1\right)^{\alpha}+\left(2-q_{i}\right)^{\alpha}\right] \log \left\{\frac{\left(2-q_{i}\right)^{\alpha}}{\left(q_{i}-1\right)^{\alpha}+\left(2-q_{i}\right)^{\alpha}}\right\}} \\
& -\alpha^{2}(\beta-1) \sum_{i=1}^{n} q_{i}^{(\lambda)} q_{i}^{(\theta)}\left\{\frac{\left(2-q_{i}\right)^{-1}\left(q_{i}-1\right)^{\alpha-1}}{\left[\left(q_{i}-1\right)^{\alpha}+\left(2-q_{i}\right)^{\alpha}\right] \log \left\{\frac{\left(2-q_{i}\right)^{\alpha}}{\left(q_{i}-1\right)^{\alpha}+\left(2-q_{i}\right)^{\alpha}}\right\}}\right\}^{2},
\end{aligned}
$$




$$
\begin{aligned}
& J_{\theta \theta}=\frac{n \lambda}{\theta^{2}}-\frac{\lambda}{\theta^{2}} \sum_{i=1}^{n}\left(\frac{x_{i}}{\theta}\right)^{2 \lambda}-2\left(\frac{\lambda}{\theta}\right)^{2} \sum_{i=1}^{n}\left(\frac{x_{i}}{\theta}\right)^{2 \lambda} \\
& +(\alpha-1) \sum_{i=1}^{n} \frac{q_{i}^{(\theta \theta)}\left(q_{i}-1\right)-\left[q_{i}^{(\theta)}\right]^{2}}{\left(q_{i}-1\right)^{2}} \\
& -(\alpha-1) \sum_{i=1}^{n} \frac{q_{i}^{(\theta \theta)}\left(2-q_{i}\right)+\left[q_{i}^{(\theta)}\right]^{2}}{\left(2-q_{i}\right)^{2}} \\
& -2 \alpha \sum_{i=1}^{n} q_{i}^{(\theta \theta)} \frac{\left(q_{i}-1\right)^{\alpha-1}-\left(2-q_{i}\right)^{\alpha-1}}{\left(q_{i}-1\right)^{\alpha}+\left(2-q_{i}\right)^{\alpha}} \\
& -2 \alpha(\alpha-1) \sum_{i=1}^{n}\left[q_{i}^{(\theta)}\right]^{2} \frac{\left(q_{i}-1\right)^{\alpha-2}+\left(2-q_{i}\right)^{\alpha-2}}{\left(q_{i}-1\right)^{\alpha}+\left(2-q_{i}\right)^{\alpha}} \\
& +2 \alpha^{2} \sum_{i=1}^{n}\left[q_{i}^{(\theta)} \frac{\left(q_{i}-1\right)^{\alpha-1}-\left(2-q_{i}\right)^{\alpha-1}}{\left(q_{i}-1\right)^{\alpha}+\left(2-q_{i}\right)^{\alpha}}\right]^{2} \\
& -\alpha(\beta-1) \sum_{i=1}^{n} \frac{\left(2-q_{i}\right)^{-1}\left(q_{i}-1\right)^{\alpha-2}\left[q_{i}^{(\theta \theta)}\left(q_{i}-1\right)+(\alpha-1)\left[q_{i}^{(\theta)}\right]^{2}\right]}{\left[\left(q_{i}-1\right)^{\alpha}+\left(2-q_{i}\right)^{\alpha}\right] \log \left\{\frac{\left(2-q_{i}\right)^{\alpha}}{\left(q_{i}-1\right)^{\alpha}+\left(2-q_{i}\right)^{\alpha}}\right\}} \\
& +\alpha^{2}(\beta-1) \sum_{i=1}^{n}\left[q_{i}^{(\theta)}\right]^{2} \frac{\left(2-q_{i}\right)^{-1}\left(q_{i}-1\right)^{\alpha-1}\left[\left(q_{i}-1\right)^{\alpha-1}-\left(2-q_{i}\right)^{\alpha-1}\right]}{\left[\left(q_{i}-1\right)^{\alpha}+\left(2-q_{i}\right)^{\alpha}\right]^{2} \log \left\{\frac{\left(2-q_{i}\right)^{\alpha}}{\left(q_{i}-1\right)^{\alpha}+\left(2-q_{i}\right)^{\alpha}}\right\}} \\
& -\alpha(\beta-1) \sum_{i=1}^{n}\left[q_{i}^{(\theta)}\right]^{2} \frac{\left(2-q_{i}\right)^{-2}\left(q_{i}-1\right)^{\alpha-1}}{\left[\left(q_{i}-1\right)^{\alpha}+\left(2-q_{i}\right)^{\alpha}\right] \log \left\{\frac{\left(2-q_{i}\right)^{\alpha}}{\left(q_{i}-1\right)^{\alpha}+\left(2-q_{i}\right)^{\alpha}}\right\}} \\
& \text { - } \alpha^{2}(\beta-1) \sum_{i=1}^{n}\left\{q_{i}^{(\theta)} \frac{\left(2-q_{i}\right)^{-1}\left(q_{i}-1\right)^{\alpha-1}}{\left[\left(q_{i}-1\right)^{\alpha}+\left(2-q_{i}\right)^{\alpha}\right] \log \left\{\frac{\left(2-q_{i}\right)^{\alpha}}{\left(q_{i}-1\right)^{\alpha}+\left(2-q_{i}\right)^{\alpha}}\right\}}\right\}^{2},
\end{aligned}
$$

where,

$$
\begin{gathered}
q_{i}^{(\lambda \lambda)}=\frac{\partial^{2} q_{i}}{\partial \lambda \partial \lambda}=2\left(\frac{x_{i}}{\theta}\right)^{\lambda}\left[1-\left(\frac{x_{i}}{\theta}\right)^{2 \lambda}\right]\left[\log \left(\frac{x_{i}}{\theta}\right)\right]^{2} \phi\left[\left(\frac{x_{i}}{\theta}\right)^{\lambda}\right] \\
q_{i}^{(\lambda \theta)}=\frac{\partial^{2} q_{i}}{\partial \lambda \partial \theta}=-\frac{2}{\theta}\left(\frac{x_{i}}{\theta}\right)^{\lambda}\left[\lambda+1-\left(\frac{x_{i}}{\theta}\right)^{2 \lambda}\right] \log \left(\frac{x_{i}}{\theta}\right) \phi\left[\left(\frac{x_{i}}{\theta}\right)^{\lambda}\right], \\
q_{i}^{(\theta \theta)}=\frac{\partial^{2} q_{i}}{\partial \theta \partial \theta}=\frac{2 \lambda}{\theta^{2}}\left(\frac{x_{i}}{\theta}\right)^{\lambda}\left[\lambda+1-\lambda\left(\frac{x_{i}}{\theta}\right)^{2 \lambda}\right] \phi\left[\left(\frac{x_{i}}{\theta}\right)^{\lambda}\right] .
\end{gathered}
$$

\section{REFERENCES}

1. K. Aas, and I. Haff, The generalized hyperbolic skew student's t-distribution, Journal of Financial Econometrics, vol. 4, pp. 275-309, 2006.

2. C. Alexander, G. M. Cordeiro, E. M. M. Ortega, and J. M. Sarabia, Generalized beta-Generated distributions, Computational Statistics and Data Analysis, vol. 56, pp. 1880-1897, 2012.

3. M. Alizadeh, M. Emadi, M. Doostparast, G. M. Cordeiro, E. M. M. Ortega, and R. R. Pescim, A new family of distributions: the Kumaraswamy odd log-logistic, properties and applications, Hacettepe Journal of Mathematics and Statistics, vol. 44, pp. 14911512,2015 
4. T. W. Anderson, and D. A. Darling, Asymptotic theory of certain" goodness of fit" criteria based on stochastic processes , The annals of mathematical statistics, pp. 193-212, 1952.

5. K. Choi, and W. Bulgren, An estimation procedure for mixtures of distributions, Journal of the Royal Statistical Society. Series B (Methodological), pp. 444-460, 1968.

6. K. Cooray, and M. M. Ananda, A generalization of the half-normal distribution with applications to lifetime data, Communications in Statistics-Theory and Methods, vol. 37(9), pp. 1323-1337, 2008.

7. G. M. Cordeiro, and M. de Castro, A new family of Generalized distributions, Journal of Statistical Computation and Simulation, vol. 81, pp. 883-898, 2011.

8. G. M. Cordeiro, R. R. Pescim, and E. M. Ortega, The Kumaraswamy generalized half-normal distribution for skewed positive data, Journal of Data Science, vol. 10(2), pp. 195-224, 2012.

9. G. M. Cordeiro, S. Nadarajah, and E. M. M. Ortega, General results for the beta Weibull distribution, Journal of Statistical Computation and Simulation, vol. 83, pp. 1082-1114, 2013.

10. G. M. Cordeiro, M. Alizadeh, E. M. Ortega, and L. H. V. Serrano, The Zografos-Balakrishnan odd log-logistic family of distributions: Properties and applications, Hacettepe Journal of Mathematics and Statistics, vol. 45(6), pp. 1781-1803, 2016.

11. G. M. Cordeiro, M. Alizadeh, R. R. Pescim, and E. M. Ortega, The odd log-logistic generalized half-normal lifetime distribution: Properties and applications, Communications in Statistics-Theory and Methods, vol. 46(9), pp. 4195-4214, $2017 \mathrm{a}$.

12. G. M. Cordeiro, M. Alizadeh, G. Ozel, B. Hosseini, E. M. M. Ortega, and E. Altun, The generalized odd log-logistic family of distributions: properties, regression models and applications, Journal of Statistical Computation and Simulation, vol. 87(5), pp. 908-932, 2017b.

13. S. Dey, J. Mazucheli and S. Nadarajah, Kumaraswamy distribution: different methods of estimation, Computational and Applied Mathematics, pp. 1-18, 2017.

14. N. Eugene, C. Lee, and F. Famoye, Beta-normal distribution and its applications, Communications in Statistics-Theory and Methods, vol. 31, pp. 497-512, 2002.

15. H. Exton, Handbook of Hypergeometric Integrals: Theory, Applications, Tables, Computer Programs, Academic Press, New York, 1978.

16. J. U. Gleaton, and J. D. Lynch, Properties of generalized log-logistic families of lifetime distributions, Journal of Probability and Statistical Science, vol. 4(1), pp. 51-64, 2006.

17. I. S. Gradshteyn, and I. M. Ryzhik, Table of Integrals, Series, and Products, sixth edition, Academic Press, San Diego, 2000.

18. B. E. Hansen, Autoregressive conditional density estimation, International Economic Review, vol. 35, pp. 705-730, 1994. (1994).

19. N. L. Johnson, S. Kotz, N. Balakrishnan, Continuous Univariate Distributions, Volume 1, 2nd edition. John Wiley and Sons, New York, 1994.

20. N. L. Johnson, S. Kotz, N. Balakrishnan, Continuous Univariate Distributions, Volume 2, 2nd edition. John Wiley and Sons, New York, 1995.

21. D. Kundu, N. Kannan, and N. Balakrishnan, On the hazard function of Birnbaum-Saunders distribution and associated inference , Computational Statistics and Data Analysis, vol. 52, pp. 2692-2702, 2008.

22. S. Nadarajah, G. M. Cordeiro and E. M. Ortega, The Zografos Balakrishnan-G family of distributions, Mathematical properties and applications, Communications in Statistics-Theory and Methods, vol. 44(1), pp. 186-215, 2015.

23. R. R. Pescim, C. G. Demtrio, G. M. Cordeiro, E. M. Ortega, and M. R. Urbano, The beta generalized half-normal distribution, Computational statistics and data analysis, vol. 54(4), pp. 945-957, 2010.

24. M. M. Ristic, and N. Balakrishnan, The gamma exponentiated exponential distribution, Journal of Statistical Computation and Simulation, pp. 1191-1206, 2012.

25. R. L. Smith, and J. C. Naylor, A comparison of maximum likelihood and Bayesian estimators for the three-parameter Weibull distribution, Journal of Applied Statistics, vol. 36, pp. 358-369, 1987.

26. J. J. Swain, S. Venkatraman, and J. R. Wilson, Least-squares estimation of distribution functions in johnson's translation system, Journal of Statistical Computation and Simulation, vol. 29(4), pp. 271-297, 1988.

27. M. Trott, The Mathematica guidebook for numerics, Springer Science and Business Media, 2009.

28. K. Zografos, and N. Balakrishnan, on families of beta- and generalized gamma-generated distributions and associated inference, Statistical Methodology, vol. 6, pp. 344-362, 2009. (2009). 